\title{
Fine behavior of functions whose gradients are in an Orlicz space
}

\author{
by \\ Jan MalÝ (Praha), David Swanson (Louisville, KY) and \\ William P. Ziemer (Bloomington, IN)
}

\begin{abstract}
For functions whose derivatives belong to an Orlicz space, we develop their "fine" properties as a generalization of the treatment found in [MZ] for Sobolev functions. Of particular importance is Theorem 8.8, which is used in the development in [MSZ] of the coarea formula for such functions.
\end{abstract}

1. Introduction. In this paper a theory of capacity is developed for functions whose derivatives are in an Orlicz space; the theory is analogous to the well-known development for classical Sobolev spaces (cf. [FZ], [Ma2], $[\mathrm{MH}],[\mathrm{Me}])$. One of our main results, Theorem 8.8, is needed in [MSZ] to find sharp conditions for the validity of the coarea formula. It is essential for this application that Orlicz-Sobolev spaces are considered rather than the ordinary Sobolev spaces.

During the preparation of this manuscript, in a survey article [M2], part of the results of this work have been announced and the methods outlined. For related results in the framework of metric spaces see also [M3].

The outline of the paper is as follows. Section 2 includes preliminaries and basic properties of Young functions and Orlicz spaces. Capacity for Orlicz-Sobolev spaces is introduced in Section 3, where it is shown that our capacity is a "true capacity" in the sense of Brelot-Choquet. The main result of Section 4 is Theorem 4.6, which states that if the Young function $\boldsymbol{F}$ satisfies a doubling condition, then functions in the corresponding Orlicz spaces have Lebesgue points everywhere except for a capacity null set. In Section 5, it is shown that Theorem 4.6 can be improved in the sense that the integrand in the expression for a Lebesgue point can be replaced by one involving a more demanding growth condition. Section 6 establishes

2000 Mathematics Subject Classification: 46E30, 46E35.

Key words and phrases: Orlicz-Sobolev space, capacity, Lebesgue points.

The research of Jan Malý has been supported by the research project MSM 0021620839 of the Czech Ministry MŠMT and by the grant GAČR 201/03/0931. 
some basic properties of capacitary extremals, while Section 7 shows how a Young function can be modified to improve its growth properties while still maintaining a particular growth property (Corollary 7.4). A fundamental inequality relating Hausdorff content and capacity (Theorem 8.8) is proved in Section 8. The last section, Section 9, shows that Lorentz spaces can be written as the union of Orlicz spaces.

There exists a parallel development of the theory which has been recently obtained by other authors. Fiorenza and Prignet [FP] obtained the comparison of capacities and Hausdorff contents, and Aïssaoui [Aï] proved that Bessel potentials of functions from Orlicz spaces have Lebesgue points q.e. measured by the corresponding capacity. A general development of Orlicz capacities defined on metric spaces has been given in $[\mathrm{BO}]$ and $[\mathrm{T}]$. However, our exposition is completely different. Aïssaoui studies capacities defined by means of convolution kernels (let us call them "potential-theoretic capacities"). In [FP], variational capacity corresponding to Orlicz-Sobolev spaces is introduced, it is shown to be equivalent to the potential-theoretic capacity and the rest is derived using potential-theoretic methods. In our work, we use intrinsic methods of variational capacity as in [MZ].

2. Preliminaries. The open ball in $\mathbb{R}^{n}$ with center $x$ and radius $r$ will be denoted by $B(x, r)$ and its volume by $\boldsymbol{\alpha}(n)$. The Lebesgue measure of a set $E$ is denoted by $|E|$ and we shall write

$$
\bar{f}_{E}:=f_{E} f d x=\frac{1}{|E|} \int_{E} f d x
$$

for the integral average of a measurable function over $E$. Whenever $\Omega \subset \mathbb{R}^{n}$ is an open set we write $\mathcal{D}(\Omega)$ for the space of all $C^{\infty}$ functions defined on $\Omega$ with compact support. We denote the integers by $\mathbb{Z}$.

Definition 2.1. Given an open set $\Omega \subset \mathbb{R}^{n}$ and $1 \leq p \leq \infty$ we denote by $W^{1, p}(\Omega)$ the Sobolev space consisting of those functions $u \in L^{p}(\Omega)$ whose first order distributional partial derivatives are also members of $L^{p}(\Omega)$. The space $W^{1, p}(\Omega)$ is a Banach space with respect to the norm

$$
\|u\|_{W^{1, p}(\Omega)}:=\|u\|_{L^{p}(\Omega)}+\|\nabla u\|_{L^{p}(\Omega)} .
$$

$W_{0}^{1, p}(\Omega)$ is the closure of $\mathcal{D}(\Omega)$ in the $W^{1, p}$ norm. $W_{\text {loc }}^{1, p}(\Omega)$ consists of those measurable functions $u$ defined on $\Omega$ which belong to $W^{1, p}(U)$ for every open set $U$ compactly contained in $\Omega$.

Definition 2.2. A nondecreasing left-continuous convex function $\boldsymbol{F}$ : $[0, \infty) \rightarrow[0, \infty]$ which satisfies $\boldsymbol{F}(0)=\lim _{t \rightarrow 0^{+}} \boldsymbol{F}(t)=0$ and $\lim _{t \rightarrow \infty} \boldsymbol{F}(t)$ $=\infty$ is called a Young function. 
A finite-valued Young function $\boldsymbol{F}$ is locally Lipschitz and has left- and right-sided derivatives which coincide except possibly on a countable set. For definiteness, throughout the paper $\boldsymbol{F}^{\prime}$ will denote the right-sided derivative of $\boldsymbol{F}$. For $t>0, \boldsymbol{F}$ admits the representation

$$
\boldsymbol{F}(t)=\int_{0}^{t} \boldsymbol{F}^{\prime}(s) d s .
$$

Definition 2.3. For $s \in[0, \infty)$, the function

$$
\widetilde{\boldsymbol{F}}(s):=\sup \{t s-\boldsymbol{F}(t): t \geq 0\}
$$

is called the complementary function to $\boldsymbol{F}$. It has the same properties as $\boldsymbol{F}$ and in addition satisfies Young's inequality

$$
t s \leq \boldsymbol{F}(t)+\widetilde{\boldsymbol{F}}(s) \quad \text { for all } t, s \geq 0 .
$$

Notice that the complementary function to $\widetilde{\boldsymbol{F}}$ is $\boldsymbol{F}$.

Definition 2.4. A Young function $\boldsymbol{F}$ is said to satisfy condition $\Delta_{2}$ globally if there exists $\boldsymbol{d}>1$ such that

$$
\boldsymbol{F}(2 t) \leq \boldsymbol{d} \boldsymbol{F}(t) \quad \text { for all } t>0 .
$$

Definition 2.5. A Young function $\boldsymbol{F}$ is said to satisfy condition $\nabla_{2}$ globally if there exists $l>1$ such that

$$
\boldsymbol{F}(t) \leq \frac{1}{2 l} \boldsymbol{F}(l t) \quad \text { for all } t>0 .
$$

The $\Delta_{2}$ condition (or $\nabla_{2}$ condition, respectively) without any modifier is usually reserved for the situation where (2.2) (or (2.3)) holds only for $t>t_{0}$ with some $t_{0}>0$. We will not use these nonglobal conditions.

Proposition 2.6. Let $\boldsymbol{F}$ be a Young function. Then

(i) $\boldsymbol{F}$ satisfies $\Delta_{2}$ globally if and only if there exists $a>1$ such that

$$
\boldsymbol{F}^{\prime}(2 t) \leq a \boldsymbol{F}^{\prime}(t) \quad \text { for all } t>0 .
$$

(ii) $\boldsymbol{F}$ satisfies $\nabla_{2}$ globally if and only if there exists $b>1$ such that

$$
2 \boldsymbol{F}^{\prime}(t) \leq \boldsymbol{F}^{\prime}(b t) \quad \text { for all } t>0 .
$$

(iii) $\boldsymbol{F}$ satisfies $\Delta_{2}$ globally if and only if there exists $p>1$ such that

$$
\frac{t \boldsymbol{F}^{\prime}(t)}{\boldsymbol{F}(t)} \leq p \quad \text { for all } t>0 .
$$

(iv) $\boldsymbol{F}$ satisfies $\nabla_{2}$ globally if and only if there exists $q>1$ such that

$$
\frac{t \boldsymbol{F}^{\prime}(t)}{\boldsymbol{F}(t)} \geq q \quad \text { for all } t>0 .
$$


Proof. Assertions (i) and (ii) follow from definitions. The remaining parts are proven in Theorem 3 on p. 22 of [RR].

Notice that (iii) and (iv) imply "stronger" $\Delta_{2}$ and $\nabla_{2}$ conditions:

$$
\begin{aligned}
& t \geq 0, l>1 \Rightarrow \boldsymbol{F}(l t) \leq l^{p} \boldsymbol{F}(t) \text { if } \boldsymbol{F} \text { is global } \Delta_{2}, \\
& t>0, l>1 \Rightarrow \boldsymbol{F}(l t) \geq l^{q} \boldsymbol{F}(t) \text { if } \boldsymbol{F} \text { is global } \nabla_{2} .
\end{aligned}
$$

These are derived by integrating

$$
\frac{\boldsymbol{F}^{\prime}(t)}{\boldsymbol{F}(t)} \leq \frac{p}{t} \quad \text { or } \quad \frac{\boldsymbol{F}^{\prime}(t)}{\boldsymbol{F}(t)} \geq \frac{q}{t}
$$

over $[t, l t]$.

Definition 2.7. Let $\Omega \subset \mathbb{R}^{n}$ and let $\boldsymbol{F}$ be a Young function. The Orlicz space $L^{\boldsymbol{F}}(\Omega)$ is the set of all measurable functions $u$ defined on $\Omega$ that satisfy

$$
\int_{\Omega} \boldsymbol{F}(|u(x)| / t) d x<\infty
$$

for some $t>0$. The space $L^{\boldsymbol{F}}$ becomes a Banach space when equipped with the Luxemburg norm

$$
\|u\|_{\boldsymbol{F} ; \Omega}:=\inf \left\{t>0: \int_{\Omega} \boldsymbol{F}(|u(x)| / t) d x \leq 1\right\} .
$$

Proposition 2.8. If $\boldsymbol{F}$ satisfies $\Delta_{2}$ globally, then

$$
u \in L^{\boldsymbol{F}}(\Omega) \quad \text { if and only if } \int_{\Omega} \boldsymbol{F}(|u(x)|) d x<\infty .
$$

Proposition 2.9. If $u \in L^{\boldsymbol{F}}(\Omega)$ and $v \in L^{\widetilde{\boldsymbol{F}}}(\Omega)$ then

$$
\int_{\Omega} u v d x \leq 2\|u\|_{\boldsymbol{F} ; \Omega}\|v\|_{\widetilde{\boldsymbol{F}} ; \Omega} .
$$

Definition 2.10. Let $\Omega$ be an open set. The Orlicz-Sobolev space $W^{1, \boldsymbol{F}}(\Omega)$ consists of all functions $u \in L^{\boldsymbol{F}}(\Omega)$ whose distributional partial derivatives are also elements of $L^{\boldsymbol{F}}(\Omega)$. The space $W^{1, \boldsymbol{F}}(\Omega)$ is a Banach space with respect to the norm

$$
\|u\|_{W^{1, \boldsymbol{F}}(\Omega)}:=\|u\|_{\boldsymbol{F} ; \Omega}+\|\nabla u\|_{\boldsymbol{F} ; \Omega} .
$$

The space $W_{0}^{1, \boldsymbol{F}}(\Omega)$ is the closure of $\mathcal{D}(\Omega)$ in the $W^{1, \boldsymbol{F}}$ norm.

Since $\boldsymbol{F}$ is convex, there exist numbers $a>0$ and $b$ such that $\boldsymbol{F}(x) \geq$ $a x+b$ for all $x \in \mathbb{R}$. Thus if $u \in L^{\boldsymbol{F}}(\Omega)$, then

$$
\int_{\Omega^{\prime}}(a|u|+b) d x \leq \int_{\Omega^{\prime}} \boldsymbol{F}(|u|) d x<\infty
$$

for every $\Omega^{\prime} \subset \subset \Omega$. In particular,

$$
W^{1, \boldsymbol{F}}(\Omega) \subset W_{\mathrm{loc}}^{1,1}(\Omega) .
$$


We will require the following results regarding the space $W^{1, \boldsymbol{F}}$. Recall from [MZ, p. 5] the definition of regularizations of a function $u \in L_{\mathrm{loc}}^{1}(\Omega)$.

Proposition 2.11 (see Adams [A]). If $\boldsymbol{F}$ satisfies $\Delta_{2}$ globally and $u \in$ $W^{1, \boldsymbol{F}}\left(\mathbb{R}^{n}\right)$, then the regularizations $u_{\varepsilon}$ converge to $u$ in $W^{1, \boldsymbol{F}}\left(\mathbb{R}^{n}\right)$. Thus

$$
W^{1, \boldsymbol{F}}\left(\mathbb{R}^{n}\right)=W_{0}^{1, \boldsymbol{F}}\left(\mathbb{R}^{n}\right) .
$$

Proposition 2.12. Suppose $\boldsymbol{F}$ satisfies $\Delta_{2}$ globally and let $B=B(x, r)$. If $u \in W^{1, \boldsymbol{F}}(B)$, then there exist $v \in W^{1, \boldsymbol{F}}\left(\mathbb{R}^{n}\right)$ and $C=C(n, \boldsymbol{d})$ such that $v=u$ on $B$, spt $v \subset B(x, 3 r / 2)$, and

$$
\begin{aligned}
\int_{\mathbb{R}^{n}} \boldsymbol{F}(|\nabla v|) d y & \leq C \int_{B} \boldsymbol{F}(|\nabla u|)+\boldsymbol{F}(|u| / r) d y, \\
\int_{\mathbb{R}^{n}} \boldsymbol{F}(|v|) d y & \leq C \int_{B} \boldsymbol{F}(|u|) d y .
\end{aligned}
$$

Proof. Assume $x=0$. Define $w$ by

$$
w(y)= \begin{cases}(2|y| / r-1) u(y) & \text { for }|y| \geq r / 2 \\ 0 & \text { for }|y|<r / 2\end{cases}
$$

and observe that

$$
|\nabla w(y)| \leq|\nabla u(y)|+2 r^{-1}|u(y)|
$$

for a.e. $y \in B(0, r) \backslash B(0, r / 2)$. Hence, by the $\Delta_{2}$ condition,

$$
\int_{B(0, r)} \boldsymbol{F}(|\nabla w|) d y \leq \int_{B(0, r)} \boldsymbol{F}(|\nabla u|) d y+\boldsymbol{d} \int_{B(0, r)} \boldsymbol{F}(|u| / r) d y .
$$

Now define $T:\{y: r \leq|y| \leq 3 r / 2\} \rightarrow\{y: r / 2 \leq|y| \leq r\}$ by

$$
T(y)=(2 r /|y|-1) y
$$

and note that $\|D T\|=1$ and the Jacobian determinant $J T$ satisfies $|J T| \geq$ $3^{1-n}$. Let

$$
v(y)= \begin{cases}0 & \text { for }|y|>3 r / 2 \\ w \circ T(y) & \text { for } r \leq|y| \leq 3 r / 2 \\ u(y) & \text { for }|y|<r\end{cases}
$$

Then, with $z:=w \circ T$,

$$
\begin{aligned}
\int_{\{r \leq|y| \leq 3 r / 2\}} \boldsymbol{F}(|\nabla z|) d y & =\int_{\{r \leq|y| \leq 3 r / 2\}} \boldsymbol{F}(|\nabla(w \circ T)|) d y \\
& \leq 3^{n-1} \int_{\{r \leq|y| \leq 3 r / 2\}} \boldsymbol{F}(|\nabla(w \circ T)|)|J T| d y \\
& \leq 3^{n-1} \int_{\{r / 2 \leq|y| \leq r\}} \boldsymbol{F}(|\nabla w|) d y
\end{aligned}
$$


and therefore, by (2.9),

$$
\begin{aligned}
\int_{B(0,3 r / 2)} \boldsymbol{F}(|\nabla v|) d y & \leq \int_{\{r \leq|y| \leq 3 r / 2\}} \boldsymbol{F}(|\nabla z|) d y+\int_{B(0, r)} \boldsymbol{F}(|\nabla u|) d y \\
& \leq 3^{n-1} \int_{\{r / 2 \leq|y| \leq r\}} \boldsymbol{F}(|\nabla w|) d y+\int_{B(0, r)} \boldsymbol{F}(|\nabla u|) d y \\
& \leq C \int_{B(0, r)} \boldsymbol{F}(|\nabla u|)+\boldsymbol{F}(|u| / r) d y .
\end{aligned}
$$

A similar argument shows that

$$
\int_{\mathbb{R}^{n}} \boldsymbol{F}(|v|) d y \leq C \int_{B} \boldsymbol{F}(|u|) d y
$$

The following Poincaré inequality is well known, but the proof is so simple that we include it here.

Proposition 2.13 (Poincaré inequality). Suppose that $\boldsymbol{F}$ satisfies $\Delta_{2}$ globally and that $\Omega$ is an open set of finite measure. There is a constant $C=C(n, \boldsymbol{F},|\Omega|)$ such that

$$
\int_{\Omega} \boldsymbol{F}(|u|) d x \leq C \int_{\Omega} \boldsymbol{F}(|\nabla u|) d x \quad \text { for all } u \in W_{0}^{1, \boldsymbol{F}}(\Omega) .
$$

Proof. In light of Proposition 2.8 we may assume that $u$ is smooth and has compact support. By the classical $W_{0}^{1,1}(\Omega)$-Poincaré inequality (cf. [Z, Theorem 2.1.11]) applied to $\boldsymbol{F}(|u|)$ (which is Lipschitz and thus in $W_{0}^{1,1}(\Omega)$ ),

$$
\int_{\Omega} \boldsymbol{F}(|u|) d x \leq C \int_{\Omega} \boldsymbol{F}^{\prime}(|u|)|\nabla u| d x .
$$

Given $\varepsilon>0$, we distinguish $|\nabla u| \leq \varepsilon|u|$ and $|\nabla u|>\varepsilon|u|$ and obtain

$$
\int_{\Omega} \boldsymbol{F}(|u|) d x \leq C(n) \int_{\Omega} \varepsilon|u| \boldsymbol{F}^{\prime}(|u|) d x+\int_{\Omega}|\nabla u| \boldsymbol{F}^{\prime}(|\nabla u| / \varepsilon) d x .
$$

From Proposition 2.6 and (2.4) we infer

$$
\int_{\Omega} \boldsymbol{F}(|u|) d x \leq p C(n) \varepsilon \int_{\Omega} \boldsymbol{F}(|u|) d x+p C(n) C(\boldsymbol{d}, \varepsilon) \int_{\Omega} \boldsymbol{F}(|\nabla u|) d x .
$$

This for $\varepsilon p C(n)<1$ yields the required inequality.

A similar argument may be used to prove other versions of the Poincaré inequality. The following Poincaré-type inequality was proven in [BL] (see also the appendix of $[\mathrm{CFL}])$.

Proposition 2.14. Let $B=B(z, r)$. If $\boldsymbol{F}$ is a Young function, then there exists $C=C(n)$ such that

$$
\int_{B} \boldsymbol{F}\left(\left|u-\bar{u}_{B}\right| / r\right) d x \leq C \int_{B} \boldsymbol{F}(|\nabla u|) d x \quad \text { for any } u \in W^{1, \boldsymbol{F}}(B) .
$$


Definition 2.15. A strictly increasing Young function $\boldsymbol{F}$ satisfying

$$
\lim _{t \rightarrow 0^{+}} \frac{\boldsymbol{F}(t)}{t}=0 \text { and } \lim _{t \rightarrow \infty} \frac{\boldsymbol{F}(t)}{t}=\infty
$$

is called an $N$-function.

A Young function is an $N$-function if and only if

$$
\boldsymbol{F}^{\prime}(0)=0, \quad 0<\boldsymbol{F}^{\prime}(t)<\infty \quad \text { for all } 0<t<\infty, \quad \lim _{t \rightarrow \infty} \boldsymbol{F}^{\prime}(t)=\infty .
$$

Proposition 2.16. A Young function $\boldsymbol{F}$ satisfying $\Delta_{2}$ and $\nabla_{2}$ globally is an $N$-function.

Proof. From (2.5) with $q>1$ we obtain the inequalities

$$
\frac{\boldsymbol{F}(a)}{a} \leq a^{q-1} \frac{\boldsymbol{F}(b)}{b} \text { and } \frac{\boldsymbol{F}(b)}{b} \geq b^{q-1} \frac{\boldsymbol{F}(a)}{a} .
$$

For $b=1$ and $a=1$, respectively, this implies

$$
\begin{array}{rlrl}
\frac{\boldsymbol{F}(a)}{a} & \leq a^{q-1} \boldsymbol{F}(1), & & a<1, \\
\frac{\boldsymbol{F}(b)}{b} & \geq b^{q-1} \boldsymbol{F}(1), & b>1 .
\end{array}
$$

The $\Delta_{2}$ condition ensures that $\boldsymbol{F}^{\prime}(t)>0$ for all $t>0$.

Definition 2.17. The inverse of a nondecreasing function $v:[0, \infty) \rightarrow$ $[0, \infty)$ satisfying $v(0)=0$ is given by

$$
v^{-1}(0)=0, \quad v^{-1}(t)=\sup \{s>0: v(s) \leq t\} \quad \text { for } t>0 .
$$

The function $v^{-1}$ is nondecreasing and satisfies $v^{-1}(v(t)) \geq t$ for all $t$. If $v$ is right-continuous then $v\left(v^{-1}(t)\right) \geq t$, and if $v$ satisfies a doubling condition of the form $v(2 t) \leq C v(t)$ for all $t>0$, then in addition we have

$$
v\left(v^{-1}(t)\right) \leq C t,
$$

where the constant $C$ is the same as the doubling constant.

3. Capacity. In this section we introduce a variational capacity in a generality which includes the capacity for Orlicz-Sobolev spaces. Our treatment is based on the variational approach to capacity. The theory of variational capacity for Sobolev spaces was developed by Maz'ya starting with [Ma1] (see also [Ma2], [AH] or [MZ] for details).

Definition 3.1. We say that a sequence $\left\{u_{j}\right\}$ converges locally weakly in $L_{\text {loc }}^{1}(\Omega)$ if it converges weakly in $L^{1}\left(\Omega^{\prime}\right)$ for any $\Omega^{\prime} \subset \subset \Omega$. We use a similar definition for the space $W_{\text {loc }}^{1,1}(\Omega)$. 
Definition 3.2. Let $\boldsymbol{F}$ be a Young function, let $c \geq 0$, and let $\Omega \subset \mathbb{R}^{n}$ be an open set. According to (2.8), the Luxemburg norm associated with the functional

$$
u \mapsto \int_{\Omega}(\boldsymbol{F}(|\nabla u|)+c \boldsymbol{F}(|u|)) d x
$$

is finite for all $u \in \mathcal{D}(\Omega)$. Denote this norm by $\|\cdot\|_{\mathcal{U}}$. We make the following assumptions on $\left(\mathcal{D}(\Omega),\|\cdot\|_{\mathcal{U}}\right)$ :

- (Continuity assumption) The embedding

$$
\left(\mathcal{D}(\Omega),\|\cdot\|_{\mathcal{U}}\right) \hookrightarrow W_{\mathrm{loc}}^{1,1}(\Omega)
$$

is continuous. This enables us to identify the completion $\mathcal{U}$ of $\left(\mathcal{D}(\Omega),\|\cdot\|_{\mathcal{U}}\right)$ as a subset of $W_{\text {loc }}^{1,1}(\Omega)$.

- (Compactness assumption) Closed balls in $\mathcal{U}$ are sequentially compact with respect to local weak convergence in $W_{\text {loc }}^{1,1}(\Omega)$.

Given a set $E \subset \Omega$, we write

$$
\mathcal{Y}(E)=\{u \in \mathcal{U}: 0 \leq u \leq 1, E \subset \operatorname{int}\{u=1\}\},
$$

where $\operatorname{int} A$ is the topological interior of $A$. We define

$$
\gamma(E)=\inf \left\{\int(\boldsymbol{F}(|\nabla u|)+c \boldsymbol{F}(|u|)) d x: u \in \mathcal{Y}(E)\right\} .
$$

REMARK 3.3. The capacity that we want to study is just this corresponding to $c=1$ and $\Omega=\mathbb{R}^{n}$. However, we need slightly more generality which will allow us to use (as an auxiliary tool) also the capacity on a ball with $c=0$, which brings to us the technical advantage of absence of the lower order term. We skip the discussion of the possibility of taking $c=0$, $\Omega=\mathbb{R}^{n}$; indeed, this leads to complications whose treatment is irrelevant for our purposes.

This definition is consistent with that in [MZ] and covers the cases studied there. For example, if $\mathcal{U}=W_{0}^{1, p}(\Omega), p>1$, then $\mathcal{U}$ is reflexive and the closed balls in $\mathcal{U}$ are sequentially weakly compact in $\mathcal{U}$. Since weak convergence in $\mathcal{U}$ implies local weak convergence in $W_{\text {loc }}^{1,1}(\Omega)$, the compactness assumption in Definition 3.2 is satisfied. In later sections we will see some situations where it is possible to overcome the possible nonreflexivity of $\mathcal{U}$.

The purpose of the continuity assumption is to prevent cases like $\|u\|_{\mathcal{U}}=$ $\|\nabla u\|_{n}$ and $\Omega=\mathbb{R}^{n}$, when there is a sequence of $\mathcal{D}\left(\mathbb{R}^{n}\right)$-functions converging in the $\mathcal{U}$-norm and locally in $L^{1}$ to the constant 1 .

We recall De Giorgi's lower semicontinuity theorem, [DG].

Theorem 3.4 (De Giorgi). Let $\boldsymbol{f}(x, \zeta, \xi): \Omega \times \mathbb{R} \times \mathbb{R}^{n} \rightarrow[0, \infty)$ be measurable in $x$, continuous in $\zeta$, and convex in $\xi$. Suppose $u_{k} \rightarrow u$ in 
$L_{\mathrm{loc}}^{1}(\Omega)$ and $p_{k} \rightarrow p$ locally weakly in $L_{\mathrm{loc}}^{1}(\Omega)$. Then

$$
\int_{\Omega} \boldsymbol{f}(x, u, p) d x \leq \liminf _{k \rightarrow \infty} \int_{\Omega} \boldsymbol{f}\left(x, u_{k}, p_{k}\right) d x .
$$

For the remainder of this section we will consider the function $\boldsymbol{f}$ given by

$$
\boldsymbol{f}(\zeta, \xi)=\boldsymbol{F}(|\xi|)+c \boldsymbol{F}(|\zeta|) .
$$

THEOREM 3.5.

(i) For any set $E \subset \Omega$,

$$
\gamma(E)=\inf \{\gamma(G): G \text { open, } G \supset E\} .
$$

(ii) If $K_{1} \supset K_{2} \supset \cdots$ are compact subsets of $\Omega$, then

$$
\gamma\left(\bigcap_{i=1}^{\infty} K_{i}\right)=\lim _{i \rightarrow \infty} \gamma\left(K_{i}\right),
$$

(iii) If $E_{1}, E_{2} \subset \Omega$, then

$$
\gamma\left(E_{1} \cup E_{2}\right)+\gamma\left(E_{1} \cap E_{2}\right) \leq \gamma\left(E_{1}\right)+\gamma\left(E_{2}\right) .
$$

(iv) If $E_{1} \subset E_{2} \subset \cdots$ are arbitrary subsets of $\Omega$, then

$$
\gamma\left(\bigcup_{i=1}^{\infty} E_{i}\right)=\lim _{i \rightarrow \infty} \gamma\left(E_{i}\right) .
$$

(v) If $\left\{E_{i}\right\}$ is a sequence of arbitrary subsets of $\Omega$ then

$$
\gamma\left(\bigcup_{i=1}^{\infty} E_{i}\right) \leq \sum_{i=1}^{\infty} \gamma\left(E_{i}\right) .
$$

Proof. (i) is evident and (ii) is an easy consequence of (i).

(iii) Given $u, v \in \mathcal{U}$, let $A:=\{u \geq v\}$ and $B:=\{u<v\}$. Then

$$
\begin{aligned}
\nabla \sup \{u, v\} & =\nabla u \chi_{A}+\nabla v \chi_{B}, \\
\nabla \inf \{u, v\} & =\nabla u \chi_{B}+\nabla v \chi_{A}, \\
\int_{\Omega} \boldsymbol{f}(\sup \{u, v\}, \nabla \sup \{u, v\}) d x & =\int_{\Omega} \boldsymbol{f}\left(u \chi_{A}+v \chi_{B}, \nabla u \chi_{A}+\nabla v \chi_{B}\right) d x \\
& =\int_{A} \boldsymbol{f}(u, \nabla u) d x+\int_{B} \boldsymbol{f}(v, \nabla v) d x, \\
\int_{\Omega} \boldsymbol{f}(\inf \{u, v\}, \nabla \inf \{u, v\}) d x & =\int_{\Omega} \boldsymbol{f}\left(u \chi_{B}+v \chi_{A}, \nabla u \chi_{B}+\nabla v \chi_{A}\right) d x \\
& =\int_{B} \boldsymbol{f}(u, \nabla u) d x+\int_{A} \boldsymbol{f}(v, \nabla v) d x,
\end{aligned}
$$


and

$$
\begin{aligned}
\int_{\Omega} \boldsymbol{f}(\sup \{u, v\}, \nabla \sup \{u, v\}) d x & +\int_{\Omega} \boldsymbol{f}(\inf \{u, v\}, \nabla \inf \{u, v\}) d x \\
& =\int_{\Omega} \boldsymbol{f}(u, \nabla u) d x+\int_{\Omega} \boldsymbol{f}(v, \nabla v) d x .
\end{aligned}
$$

It follows that $\sup \{u, v\}, \inf \{u, v\} \in \mathcal{U}$, and as $u$ runs over $\mathcal{Y}\left(E_{1}\right)$ and $v$ runs over $\mathcal{Y}\left(E_{2}\right)$, and we easily obtain (iii).

(iv) Obviously $\lim _{i \rightarrow \infty} \gamma\left(E_{i}\right) \leq \gamma\left(\bigcup_{i=1}^{\infty} E_{i}\right)$. To prove the opposite inequality let $\varepsilon>0$ and choose $u_{i} \in \mathcal{Y}\left(E_{i}\right)$ such that

$$
\int_{\Omega} \boldsymbol{f}\left(u_{i}, \nabla u_{i}\right) d x \leq \gamma\left(E_{i}\right)+\frac{\varepsilon}{2^{i}} .
$$

Let $v_{j}:=\sup \left\{u_{1}, \ldots, u_{j}\right\}$ and note that

$$
v_{j}=\sup \left\{v_{j-1}, u_{j}\right\} \quad \text { and } \quad E_{j-1} \subset \operatorname{int}\left\{\inf \left\{v_{j-1}, u_{j}\right\}=1\right\}
$$

since $u_{j}, v_{j-1} \geq 1$ on a neighborhood of $E_{j-1}$. Then, using (3.3), we get

$$
\begin{aligned}
\int_{\Omega} \boldsymbol{f}\left(v_{j}, \nabla v_{j}\right) d x+\gamma\left(E_{j-1}\right) \leq & \int_{\Omega} \boldsymbol{f}\left(\sup \left\{v_{j-1}, u_{j}\right\}, \nabla \sup \left\{v_{j-1}, u_{j}\right\}\right) d x \\
& +\int_{\Omega} \boldsymbol{f}\left(\inf \left\{v_{j-1}, u_{j}\right\}, \nabla \inf \left\{v_{j-1}, u_{j}\right\}\right) d x \\
= & \int_{\Omega} \boldsymbol{f}\left(v_{j-1}, \nabla v_{j-1}\right) d x+\int_{\Omega} \boldsymbol{f}\left(u_{j}, \nabla u_{j}\right) d x \\
\leq & \int_{\Omega} \boldsymbol{f}\left(v_{j-1}, \nabla v_{j-1}\right) d x+\gamma\left(E_{j}\right)+\frac{\varepsilon}{2^{j}} .
\end{aligned}
$$

By induction, we obtain

$$
\int_{\Omega} \boldsymbol{f}\left(v_{j}, \nabla v_{j}\right) d x \leq \gamma\left(E_{j}\right)+\sum_{i=1}^{j} \frac{\varepsilon}{2^{i}}<\lim _{i \rightarrow \infty} \gamma\left(E_{i}\right)+\varepsilon
$$

for all $j$. We may assume that $\lim _{i \rightarrow \infty} \gamma\left(E_{i}\right)<\infty$. By (2.6) and (3.2) the sequence $\left\{v_{j}\right\}$ is bounded in $\mathcal{U}$. By the compactness assumption in Definition 3.2 and the compact embedding theorem, there exists a subsequence (not relabeled) of the sequence $\left\{v_{j}\right\}$ converging weakly in $W_{\mathrm{loc}}^{1,1}(\Omega)$ and strongly in $L_{\text {loc }}^{1}(\Omega)$ to a function $v \in \mathcal{U}$. By lower semicontinuity (Theorem 3.4),

$$
\int_{\Omega} \boldsymbol{f}(v, \nabla v) d x \leq \liminf _{j \rightarrow \infty} \int_{\Omega} \boldsymbol{f}\left(v_{j}, \nabla v_{j}\right) d x \leq \lim _{i \rightarrow \infty} \gamma\left(E_{i}\right)+\varepsilon .
$$

Now define $w=\lim _{j \rightarrow \infty} v_{j}$. Then $\bigcup_{i=1}^{\infty} E_{i} \subset \operatorname{int}\{w=1\}$, and since $v_{j} \rightarrow w$ almost everywhere, the weak limit of the $v_{j}$ cannot be anything other than $w$. 
Thus $v=w$ and so $v \in \mathcal{Y}\left(\bigcup_{i=1}^{\infty} E_{i}\right)$. It follows that

$$
\gamma\left(\bigcup_{i=1}^{\infty} E_{i}\right) \leq \int_{\Omega} \boldsymbol{f}(v, \nabla v) d x \leq \lim _{i \rightarrow \infty} \gamma\left(E_{i}\right)+\varepsilon .
$$

(v) By induction, from (iii) we derive

$$
\gamma\left(\bigcup_{i=1}^{k} E_{i}\right) \leq \sum_{i=1}^{k} \gamma\left(E_{i}\right)
$$

for any finite selection of sets $E_{1}, \ldots, E_{k}$. Now refer to (iv) to obtain

$$
\gamma\left(\bigcup_{i=1}^{\infty} E_{i}\right)=\lim _{k \rightarrow \infty} \gamma\left(\bigcup_{i=1}^{k} E_{i}\right) \leq \lim _{k \rightarrow \infty} \sum_{i=1}^{k} \gamma\left(E_{i}\right)=\sum_{i=1}^{\infty} \gamma\left(E_{i}\right) .
$$

Proposition 3.6. Suppose that $G \subset \Omega$ is an open set and $\mathcal{Y}(G) \neq \emptyset$. Then there exists a minimizer of

$$
\int_{\Omega}(\boldsymbol{F}(|\nabla u|)+c \boldsymbol{F}(|u|)) d x
$$

in $\mathcal{Y}(G)$.

Proof. Let $\left\{u_{j}\right\} \subset \mathcal{Y}(G)$ be a minimizing sequence for (3.7). Then $\left\{u_{j}\right\}$ is bounded in $\mathcal{U}$, and proceeding as in the proof of Theorem 3.5 we may pass to a subsequence converging weakly in $W_{\text {loc }}^{1,1}(\Omega)$ and strongly in $L_{\text {loc }}^{1}(\Omega)$ to a function $u \in \mathcal{U}$ (hence $u \geq 1$ on $G$ ). Thus

$$
\int_{\Omega}(\boldsymbol{F}(|\nabla u|)+c \boldsymbol{F}(|u|)) d x \leq \liminf _{j \rightarrow \infty} \int_{\Omega}\left(\boldsymbol{F}\left(\left|\nabla u_{j}\right|\right)+c \boldsymbol{F}\left(\left|u_{j}\right|\right)\right) d x=\gamma(G) .
$$

Since $u \geq 1$ on $G$, it follows that $u \in \mathcal{Y}(G)$ and we obtain the opposite inequality

$$
\gamma(G) \leq \int_{\Omega}(\boldsymbol{F}(|\nabla u|)+c \boldsymbol{F}(|u|)) d x,
$$

implying that $u$ is a minimizer.

Remark 3.7. The minimizer from Proposition 3.6 is often called a $c a-$ pacitary extremal for $\gamma(G)$. It is sometimes desirable to have uniqueness for capacitary extremals; this requires stronger assumptions on $\boldsymbol{F}$. For instance, strict convexity of $\boldsymbol{F}$ is sufficient to imply uniqueness.

4. Lebesgue points and Orlicz spaces. In this section we prove a generalization of the result concerning Sobolev functions and their Lebesgue points (cf. [FZ]). Namely, we show that a function in $W^{1, \boldsymbol{F}}$ has Lebesgue points q.e. for the capacity $\gamma_{\boldsymbol{F}}$. Alternatively, these results can be seen from 
those of Aïssaoui [Aï] which depend on deep results on equivalence of function spaces and capacities [FP]. We also mention quasicontinuous representatives (see $[\mathrm{AH}]$ or $[\mathrm{MZ}]$ for historical notes).

We assume throughout this section that $\boldsymbol{F}$ satisfies $\Delta_{2}$ globally with doubling constant $\boldsymbol{d}$.

Definition 4.1. Given a Young function $\boldsymbol{F}$, we define the capacity $\gamma_{\boldsymbol{F}}$ on $\mathbb{R}^{n}$ as

$$
\gamma_{\boldsymbol{F}}(E)=\inf \left\{\int_{\mathbb{R}^{n}}(\boldsymbol{F}(|u|)+\boldsymbol{F}(|\nabla u|)) d x: u \in W^{1, \boldsymbol{F}}\left(\mathbb{R}^{n}\right), E \subset \operatorname{int}\{u \geq 1\}\right\} .
$$

If $\boldsymbol{F}$ is an $N$-function, this definition is in accordance with the general definition of capacity in (3.1) with $c=1$. A function $u$ is called $\boldsymbol{F}$-quasicontinuous if for each $\varepsilon>0$ there exists an open set $U_{\varepsilon}$ such that $\gamma_{\boldsymbol{F}}\left(U_{\varepsilon}\right)<\varepsilon$ and $u$ is continuous on $\mathbb{R}^{n} \backslash U_{\varepsilon}$.

The $\Delta_{2}$ assumption and Proposition 2.11 imply

$$
\mathcal{U}=W^{1, \boldsymbol{F}}\left(\mathbb{R}^{n}\right) .
$$

The choice $c=1$ and Proposition 2.9 make the continuity assumption trivial. The compactness assumption is verified by the De La Vallée Poussin criterion for weak compactness in $L^{1}$ and Theorem 3.4. Precisely, if $\left\{u_{j}\right\}$ is a sequence of functions from a closed ball in $\mathcal{U}$, the De La Vallée Poussin criterion shows that, after passing to a subsequence, $\left\{u_{j}\right\}$ has a weak limit in $W_{\text {loc }}^{1,1}\left(\mathbb{R}^{n}\right)$, which is strong in $L_{\text {loc }}^{1}\left(\mathbb{R}^{n}\right)$ by the compact embedding theorem. The lower semicontinuity of the $\mathcal{U}$-norm then shows that the limit remains in $\mathcal{U}$.

The nondecreasing nature of $\boldsymbol{F}$ implies that $W^{1, \boldsymbol{F}}\left(\mathbb{R}^{n}\right)$ is closed under truncation and

$$
\begin{aligned}
\gamma_{\boldsymbol{F}}(E)=\inf \left\{\int_{\mathbb{R}^{n}}(\boldsymbol{F}(|u|)+\boldsymbol{F}(|\nabla u|)) d x:\right. & \\
& \left.u \in W^{1, \boldsymbol{F}}\left(\mathbb{R}^{n}\right), 0 \leq u \leq 1, E \subset \operatorname{int}\{u=1\}\right\} .
\end{aligned}
$$

Definition 4.2. If $u \in L_{\mathrm{loc}}^{1}\left(\mathbb{R}^{n}\right)$, its corresponding maximal function is defined by

$$
M u(x)=\sup _{r>0} f_{B(x, r)}|u(y)| d y
$$

for all $x \in \mathbb{R}^{n}$.

THEOREM 4.3. There exists a constant $C=C(n, \boldsymbol{d})$ such that

$$
\gamma_{\boldsymbol{F}}(\{M u>1\}) \leq C \int_{\mathbb{R}^{n}}(\boldsymbol{F}(|u|)+\boldsymbol{F}(|\nabla u|)) d x
$$

for all $u \in W^{1, \boldsymbol{F}}\left(\mathbb{R}^{n}\right)$. 
Proof. Assume without loss of generality that $u$ is nonnegative, and suppose first that $u$ has compact support. With $A:=\{x: M u(x)>1\}$ and $\bar{u}(x, r):=f_{B(x, r)} u(y) d y$, note that if $x \in A$, then $\bar{u}(x, r)>1$ for some $r>0$. For such an $r$,

$$
|B(x, r)| \leq \int_{B(x, r)} u d x \leq \int_{\operatorname{spt} u}|u| d x<\infty,
$$

so that $r$ is bounded above by some constant $C$. Consequently, we may appeal to the Besicovitch covering lemma to conclude that there exist an integer $N>1$ and a sequence $\left\{B_{j}\right\}$ of balls such that

$$
A \subset \bigcup_{j=1}^{\infty} B_{j}, \quad \sum_{j} \chi_{B_{j}} \leq N,
$$

and

$$
f_{B_{j}} u d x>1 \quad \text { for each } j=1,2, \ldots
$$

Now consider $\varphi:=\left(\bar{u}_{B_{j}}-u\right)^{+}$. Applying the $\Delta_{2}$ condition and Jensen's inequality we have

$$
\boldsymbol{F}(|\varphi|) \leq \frac{\boldsymbol{d}}{2}\left(\boldsymbol{F}\left(\left|\bar{u}_{B_{j}}\right|\right)+\boldsymbol{F}(|u|)\right) \leq \frac{\boldsymbol{d}}{2}\left(f_{B_{j}} \boldsymbol{F}(|u|) d y+\boldsymbol{F}(|u|)\right),
$$

and hence

$$
\int_{B_{j}} \boldsymbol{F}(|\varphi|) d x \leq \boldsymbol{d} \int_{B_{j}} \boldsymbol{F}(|u|) d x .
$$

Likewise, since $|\nabla \varphi| \leq|\nabla u|$, we have

$$
\int_{B_{j}} \boldsymbol{F}(|\nabla \varphi|) d x \leq \int_{B_{j}} \boldsymbol{F}(|\nabla u|) d x
$$

and so $\varphi \in W^{1, \boldsymbol{F}}\left(B_{j}\right)$. We refer to Proposition 2.12, (4.2), and Poincaré's inequality (Proposition 2.14) to obtain a function $v_{j} \in W^{1, \boldsymbol{F}}\left(\mathbb{R}^{n}\right)$ such that $v_{j}=\varphi$ on $B_{j}$ and

$$
\begin{aligned}
\int_{\mathbb{R}^{n}}\left(\boldsymbol{F}\left(\left|\nabla v_{j}\right|\right)+\boldsymbol{F}\left(v_{j}\right)\right) d x & \leq C \int_{B_{j}}(\boldsymbol{F}(|\nabla \varphi|)+\boldsymbol{F}(|\varphi| / r)+\boldsymbol{F}(|\varphi|)) d x \\
& \leq C \int_{B_{j}}\left(\boldsymbol{F}(|u|)+\boldsymbol{F}(|\nabla u|)+\boldsymbol{F}\left(\left|\bar{u}_{B_{j}}-u\right| / r\right)\right) d x \\
& \leq C \int_{B_{j}}(\boldsymbol{F}(|u|)+\boldsymbol{F}(|\nabla u|)) d x,
\end{aligned}
$$

where $C=C(n, \boldsymbol{d})$ and $r$ is the radius of $B_{j}$. Note that

$$
u+v_{j} \geq \bar{u}_{B_{j}} \geq 1 \quad \text { on } B_{j} .
$$


Therefore, defining

$$
w_{j}=\sup \left\{v_{1}, \ldots, v_{j}\right\},
$$

we see that

$$
u+w_{j}>1 \quad \text { on } A \cap\left(B_{1} \cup \cdots \cup B_{j}\right) .
$$

Note that there exist disjoint sets $A_{1}, \ldots, A_{j}$ such that

$$
\begin{aligned}
w_{j} & =\sum_{i=1}^{j} \chi_{A_{i}} v_{i}, & \nabla w_{j} & =\sum_{i=1}^{j} \chi_{A_{i}} \nabla v_{i} \quad \text { a.e., } \\
\boldsymbol{F}\left(w_{j}\right) & =\sum_{i=1}^{j} \chi_{A_{i}} \boldsymbol{F}\left(v_{i}\right), & \boldsymbol{F}\left(\nabla w_{j}\right) & =\sum_{i=1}^{j} \chi_{A_{i}} \nabla \boldsymbol{F}\left(v_{i}\right)
\end{aligned}
$$

and therefore, since $\boldsymbol{F} \in \Delta_{2}$ globally,

$$
\begin{aligned}
& \int_{\mathbb{R}^{n}}(\boldsymbol{F}\left.\left(\left|\nabla\left(u+w_{j}\right)\right|\right)+\boldsymbol{F}\left(\left|u+w_{j}\right|\right)\right) d x \\
& \leq C \int_{\mathbb{R}^{n}}(\boldsymbol{F}(|\nabla u|)+\boldsymbol{F}(|u|)) d x+C \int_{\mathbb{R}^{n}}\left(\boldsymbol{F}\left(\left|\nabla w_{j}\right|\right)+\boldsymbol{F}\left(\left|w_{j}\right|\right)\right) d x \\
& \leq C \int_{\mathbb{R}^{n}}(\boldsymbol{F}(|\nabla u|)+\boldsymbol{F}(|u|)) d x+C \sum_{i=1}^{j} \int_{A_{i}}\left(\boldsymbol{F}\left(\left|\nabla v_{i}\right|\right)+\boldsymbol{F}\left(\left|v_{i}\right|\right)\right) d x \\
& \leq C \int_{\mathbb{R}^{n}}(\boldsymbol{F}(|\nabla u|)+\boldsymbol{F}(|u|)) d x+C \sum_{i=1}^{j} \int_{B_{i}}(\boldsymbol{F}(|\nabla u|)+\boldsymbol{F}(|u|)) d x \\
& \leq C \int_{\mathbb{R}^{n}}(\boldsymbol{F}(|\nabla u|)+\boldsymbol{F}(|u|)) d x+C N \int_{\mathbb{R}^{n}}(\boldsymbol{F}(|\nabla u|)+\boldsymbol{F}(|u|)) d x .
\end{aligned}
$$

Since $A$ is open,

$$
\gamma_{\boldsymbol{F}}\left(A \cap\left(B_{1} \cup \cdots \cup B_{j}\right)\right) \leq C \int_{\mathbb{R}^{n}}(\boldsymbol{F}(|\nabla u|)+\boldsymbol{F}(|u|)) d x
$$

and taking the limit as $j \rightarrow \infty$, we obtain, by Theorem 3.5(iv),

$$
\gamma_{\boldsymbol{F}}(A) \leq C \int_{\mathbb{R}^{n}}(\boldsymbol{F}(|\nabla u|)+\boldsymbol{F}(|u|)) d x,
$$

as desired. In case $u$ fails to have compact support, apply the preceding argument to $u \eta_{m}$ where $\eta_{m}$ is a smooth cut-off function satisfying

$$
\chi_{B(0, m)} \leq \eta_{m} \leq \chi_{B(0,2 m)} \quad \text { and } \quad\left|\nabla \eta_{m}\right| \leq 2 / m .
$$

Since $\left|\nabla\left(u \eta_{m}\right)\right| \leq 2 m^{-1}|u|+|\nabla u|$, the $\Delta_{2}$ condition implies

$$
\gamma_{\boldsymbol{F}}\left(\left\{M\left(u \eta_{m}\right)>1\right\}\right) \leq C \int_{\mathbb{R}^{n}}(\boldsymbol{F}(|\nabla u|)+\boldsymbol{F}(|u|)) d x
$$


for all $m \geq 1$. Using the fact that

$$
\{M u>1\}=\bigcup_{m=1}^{\infty}\left\{M\left(u \eta_{m}\right)>1\right\}
$$

we derive the result from Theorem 3.5(iv).

COROLlaRY 4.4. Under the same hypotheses as in the previous theorem,

$$
\gamma_{\boldsymbol{F}}(\{M u>\lambda\}) \leq \frac{C}{\lambda} \int_{\mathbb{R}^{n}}(\boldsymbol{F}(|u|)+\boldsymbol{F}(|\nabla u|)) d x
$$

for all $\lambda>1$. Consequently,

$$
\gamma_{\boldsymbol{F}}(\{M u=\infty\})=0 .
$$

Proof. Assume $\lambda>1$. With $v:=u / \lambda$, the convexity of $\boldsymbol{F}$ yields

$$
\begin{aligned}
\gamma_{\boldsymbol{F}}(\{M v>1\}) & \leq C \int_{\mathbb{R}^{n}}(\boldsymbol{F}(|u / \lambda|)+\boldsymbol{F}(|\nabla u / \lambda|)) d x \\
& \leq \frac{C}{\lambda} \int_{\mathbb{R}^{n}}(\boldsymbol{F}(|u|)+\boldsymbol{F}(|\nabla u|)) d x .
\end{aligned}
$$

If $E:=\{x: M u(x)=\infty\}$, then $E \subset\{x: M u(x)>\lambda\}$ for any $\lambda>0$. Hence

$$
\gamma_{\boldsymbol{F}}(E) \leq \frac{C}{\lambda} \int_{\mathbb{R}^{n}}(\boldsymbol{F}(|u|)+\boldsymbol{F}(|\nabla u|)) d x \rightarrow 0 \quad \text { as } \lambda \rightarrow \infty .
$$

Definition 4.5. For $u \in L_{\text {loc }}^{1}(\Omega)$, for any ball $B=B(x, r)$ we will write

Further, we define

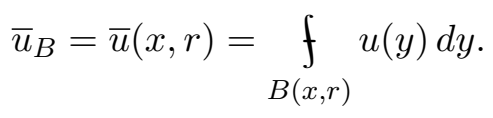

$$
\bar{u}(x):=\lim _{r \rightarrow 0^{+}} \bar{u}(x, r)
$$

whenever the limit exists. The function $\bar{u}$, which coincides almost everywhere with $u$, is called the precise representative of $u$. We say that $u$ is precisely represented if it is identified with $\bar{u}$ at all points $x$ where the above limit exists.

Theorem 4.6. If $u \in W_{\mathrm{loc}}^{1, \boldsymbol{F}}(\Omega)$, then $\bar{u}(x)$ exists and

$$
\lim _{r \rightarrow 0^{+}} f_{B(x, r)}|u(y)-\bar{u}(x)| d y=0
$$

for all $x$ except a $\gamma_{\boldsymbol{F}^{-} \text {-null set. }}$

Proof. Since the result is local, we may assume that $\Omega=\mathbb{R}^{n}$ and $u$ has compact support. Since $\boldsymbol{F} \in \Delta_{2}$, Proposition 2.11 implies that for each $\varepsilon>0$ there exists $u_{\varepsilon} \in \mathcal{D}\left(\mathbb{R}^{n}\right)$ such that

$$
\int_{\mathbb{R}^{n}}\left(\boldsymbol{F}\left(\left|u-u_{\varepsilon}\right|\right)+\boldsymbol{F}\left(\left|\nabla u-\nabla u_{\varepsilon}\right|\right)\right) d x<\varepsilon .
$$


With $\varepsilon_{k}=2^{-p k-k}$, where $p$ is as in Proposition 2.6(iii), we set

$$
E_{k}=\left\{x \in \mathbb{R}^{n}: M\left(u-u_{\varepsilon_{k}}\right)>2^{-k-1}\right\}, \quad G_{k}=\bigcup_{j \geq k} E_{j} .
$$

By Theorem 4.3, (4.5) and (2.4),

$$
\begin{aligned}
\gamma_{\boldsymbol{F}}\left(E_{k}\right) & =\gamma_{\boldsymbol{F}}\left\{x \in \mathbb{R}^{n}: M\left(2^{k}\left(u-u_{\varepsilon_{k}}\right)\right)>1\right\} \\
& \leq C \int_{\mathbb{R}^{n}}\left(\boldsymbol{F}\left(2^{k}\left|u-u_{\varepsilon_{k}}\right|\right)+\boldsymbol{F}\left(2^{k}\left|\nabla\left(u-u_{\varepsilon_{k}}\right)\right|\right)\right) d x \\
& \leq C 2^{p k} \int_{\mathbb{R}^{n}}\left(\boldsymbol{F}\left(\left|u-u_{\varepsilon_{k}}\right|\right)+\boldsymbol{F}\left(\left|\nabla\left(u-u_{\varepsilon_{k}}\right)\right|\right)\right) d x \leq C 2^{p k} \varepsilon_{k} \leq C 2^{-k}
\end{aligned}
$$

and thus by countable subadditivity also

$$
\gamma_{\boldsymbol{F}}\left(G_{k}\right) \leq C 2^{-k}
$$

Define $\chi_{r}:=|B(0, r)|^{-1} \chi_{B(0, r)}$. If $x \notin G_{k}$, for $j \geq k$ we have

$$
\begin{aligned}
|\bar{u}(x, r)-\bar{u}(x, \delta)| \leq & \left|\chi_{r} * u_{\varepsilon_{j}}(x)-\chi_{\delta} * u_{\varepsilon_{j}}(x)\right| \\
& +\left|\chi_{r} *\left(u-u_{\varepsilon_{j}}\right)(x)\right|+\left|\chi_{\delta} *\left(u-u_{\varepsilon_{j}}\right)(x)\right|
\end{aligned}
$$

and thus

$$
\limsup _{r, \delta \rightarrow 0}\left|\chi_{r} * u(x)-\chi_{\delta} * u(x)\right| \leq 2 M\left(u-u_{\varepsilon_{j}}\right)(x) \leq 2^{-j} .
$$

It follows that there exists $\bar{u}(x)=\lim _{r \rightarrow 0} \chi_{r} * u(x)$. Choosing $j \geq k$ again, we observe $\left|\bar{u}(x)-u_{\varepsilon_{j}}(x)\right| \leq M\left(u-u_{\varepsilon}\right)(x)$. Now,

$\underset{B(x, r)}{f}|u-\bar{u}(x)| d y \leq \chi_{r} *\left|u-u_{\varepsilon_{j}}\right|(x)+\chi_{r} *\left|u_{\varepsilon_{j}}-u_{\varepsilon_{j}}(x)\right|(x)+\left|u_{\varepsilon_{j}}(x)-u(x)\right|$ and hence

$$
\limsup _{r \rightarrow 0} \chi_{r} *|u-\bar{u}(x)|(x) \leq 2 M\left(u-u_{\varepsilon_{j}}\right)(x) \leq 2^{-j} .
$$

It follows that $x$ is a Lebesgue point for $\bar{u}$. Since $\gamma_{\boldsymbol{F}}\left(\bigcap_{k=1}^{\infty} G_{k}\right)=0, \bar{u}$ exists and $x$ is a Lebesgue point for $\bar{u}$ for all $x$ except a $\gamma_{\boldsymbol{F}}$-null set.

COROLlary 4.7. Under the hypotheses of the previous theorem, the function $u$ has an $\boldsymbol{F}$-quasicontinuous representative.

Proof. In the previous proof, observe that the sets $G_{k}$ are open, and for $x \in \mathbb{R}^{n} \backslash G_{k}$,

$$
\left|\bar{u}(x)-u_{\varepsilon_{j}}(x)\right| \leq M\left(u-u_{\varepsilon_{j}}\right)(x) \leq 2^{-j}
$$

for all $j \geq k$. Thus $u_{\varepsilon_{j}} \rightarrow \bar{u}$ uniformly on $\mathbb{R}^{n} \backslash G_{k}$.

Next we show how the capacity $\gamma_{\boldsymbol{F}}$ may be tested by functions outside the class $\mathcal{Y}(E)$. It follows from Theorems 4.3 and 4.6 that if $u \in W^{1, \boldsymbol{F}}\left(\mathbb{R}^{n}\right)$ 
then

$$
\gamma_{\boldsymbol{F}}(\{\bar{u} \geq 1\}) \leq C \int_{\mathbb{R}^{n}}(\boldsymbol{F}(|u|)+\boldsymbol{F}(|\nabla u|)) d x .
$$

The next result shows that this remains valid with $C=1$ and with $\bar{u}$ replaced by any $\boldsymbol{F}$-quasicontinuous representative of $u$.

Theorem 4.8. Suppose that $u \in W^{1, \boldsymbol{F}}\left(\mathbb{R}^{n}\right)$ is $\boldsymbol{F}$-quasicontinuous. If

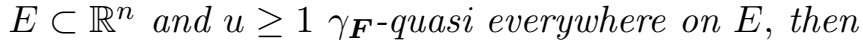

$$
\gamma_{\boldsymbol{F}}(E) \leq \int_{\mathbb{R}^{n}}(\boldsymbol{F}(|u|)+\boldsymbol{F}(|\nabla u|)) d x .
$$

Proof. Let $p$ be as in Proposition 2.6(iii). For every $k \geq 1$ choose an open set $U_{k}$ with $\gamma_{\boldsymbol{F}}\left(U_{k}\right)<2^{-k-1}$ such that $\left.u\right|_{\mathbb{R}^{n} \backslash U_{k}}$ is continuous, and an open set $V_{k}$ with $\gamma_{\boldsymbol{F}}\left(V_{k}\right)<2^{-k-1}$ such that $u \geq 1$ everywhere on $E \backslash V_{k}$. Let $W_{k}=U_{k} \cup V_{k}$. Then $\gamma_{\boldsymbol{F}}\left(W_{k}\right)<2^{-k}$, so there exists $w_{k} \in \mathcal{Y}\left(U_{k}\right)$ satisfying

$$
\int_{\mathbb{R}^{n}}\left(\boldsymbol{F}\left(\left|w_{k}\right|\right)+\boldsymbol{F}\left(\left|\nabla w_{k}\right|\right)\right) d x<2^{-k} .
$$

The set $\{u>1-1 / k\}$ is a relatively open subset of $\mathbb{R}^{n} \backslash W_{k}$ containing $E \backslash W_{k}$, so

$$
G_{k}:=W_{k} \cup\{u>1-1 / k\}
$$

is an open set containing $E$. The function

$$
v_{k}:=\frac{k}{k-1} u+w_{k}
$$

belongs to $W^{1, \boldsymbol{F}}\left(\mathbb{R}^{n}\right)$ and satisfies $v_{k} \geq 1$ on $G_{k}$, implying that

$$
\gamma_{\boldsymbol{F}}(E) \leq \gamma_{\boldsymbol{F}}\left(G_{k}\right) \leq \int_{\mathbb{R}^{n}}\left(\boldsymbol{F}\left(\left|v_{k}\right|\right)+\boldsymbol{F}\left(\left|\nabla v_{k}\right|\right)\right) d x .
$$

We have

$$
v_{k}=\frac{k}{k+1} \frac{k+1}{k-1} u+\frac{1}{k+1}(k+1) w_{k},
$$

so by convexity and (2.4),

$$
\begin{aligned}
\boldsymbol{F}\left(\left|v_{k}\right|\right)+\boldsymbol{F}\left(\left|\nabla v_{k}\right|\right) \leq & \frac{k}{k+1}\left(\boldsymbol{F}\left(\frac{k+1}{k-1}|u|\right)+\boldsymbol{F}\left(\frac{k+1}{k-1}|\nabla u|\right)\right) \\
& +\frac{1}{k+1}\left(\boldsymbol{F}\left((k+1)\left|w_{k}\right|\right)+\boldsymbol{F}\left((k+1)\left|\nabla w_{k}\right|\right)\right) \\
\leq & \frac{k}{k+1}\left(\frac{k+1}{k-1}\right)^{p}(\boldsymbol{F}(|u|)+\boldsymbol{F}(|\nabla u|)) \\
& +\frac{(k+1)^{p}}{k+1}\left(\boldsymbol{F}\left(\left|w_{k}\right|\right)+\boldsymbol{F}\left(\left|\nabla w_{k}\right|\right)\right) .
\end{aligned}
$$


It follows that

$$
\begin{aligned}
\gamma_{\boldsymbol{F}}(E) & \leq \int_{\mathbb{R}^{n}}\left(\boldsymbol{F}\left(\left|v_{k}\right|\right)+\boldsymbol{F}\left(\left|\nabla v_{k}\right|\right)\right) d x \\
& \leq \frac{(k+1)^{p-1} k}{(k-1)^{p}} \int_{\mathbb{R}^{n}}(\boldsymbol{F}(|u|)+\boldsymbol{F}(|\nabla u|)) d x+2^{-k}(k+1)^{p-1},
\end{aligned}
$$

which implies the result.

COROLlaRY 4.9. Let $u \in W^{1, \boldsymbol{F}}\left(\mathbb{R}^{n}\right)$ and suppose that $u_{1}$ and $u_{2}$ are two quasicontinuous representatives of $u$. Then $u_{1}=u_{2} \gamma_{\boldsymbol{F}}$-quasieverywhere in $\mathbb{R}^{n}$.

5. Lebesgue points - an improvement. In the standard development of Lebesgue points for functions $u \in W^{1, p}\left(\mathbb{R}^{n}\right)$, we have the following result that is an improvement of Theorem 4.6: With $1 \leq p<n$ and $p^{*}:=n p /(n-p)$,

$$
\lim _{r \rightarrow 0^{+}} \underset{B(x, r)}{f}|u(y)-u(x)|^{p^{*}} d y=0
$$

for all $x$ in the complement of a $p$-capacity null set. In this section, we establish an analogous result in the context of Orlicz-Sobolev spaces. When $\boldsymbol{F}(t)=t^{p},(5.8)$ in Theorem 5.8 below is precisely (5.1).

Lemma 5.1. Let $\boldsymbol{F}$ be a Young function satisfying $\Delta_{2}$ globally and suppose that $g \in L^{\boldsymbol{F}}\left(\mathbb{R}^{n}\right)$. Then

$$
\lim _{r \rightarrow 0^{+}} \frac{1}{\boldsymbol{F}(1 / r)} f_{B(x, r)} \boldsymbol{F}(|g|) d y=0 \quad \text { for } \gamma_{\boldsymbol{F}^{-}} \text {q.e. } x \in \mathbb{R}^{n} .
$$

Proof. For $\varepsilon>0$, consider

$$
A_{\varepsilon}=\left\{x \in \mathbb{R}^{n}: \limsup _{r \rightarrow 0^{+}} \frac{1}{\boldsymbol{F}(1 / r)} \underset{B(x, r)}{f} \boldsymbol{F}(|g|) d y>\varepsilon\right\} .
$$

Since $A_{\varepsilon}$ does not contain any Lebesgue point of $\boldsymbol{F}(g)$, it follows that $\left|A_{\varepsilon}\right|=0$. Choose an open set $G$ containing $A_{\varepsilon}$. For each $x \in A_{\varepsilon}$ we find $r(x) \in(0,1)$ such that $B(x, r(x)) \subset G$ and

$$
\frac{1}{\boldsymbol{F}(1 / r)} f_{B(x, r(x))} \boldsymbol{F}(|g|) d y>\varepsilon .
$$

Using a well-known covering theorem (cf. [Z, Theorem 1.3.1]) we can find a disjoint sequence $\left\{B_{i}\right\}$ of balls, $B_{i}=B\left(x_{i}, r_{i}\right)$, such that $r_{i}=r\left(x_{i}\right)$ and

$$
A_{\varepsilon} \subset \bigcup_{i=1}^{\infty} \widehat{B}_{i}, \quad \text { where } \quad \widehat{B}_{i}=B\left(x_{i}, 5 r_{i}\right) .
$$


For each $i$ let $\eta_{i}$ be a smooth cut-off function such that

$$
\chi_{B\left(x_{i}, 5 r_{i}\right)} \leq \eta_{i} \leq \chi_{B\left(x_{i}, 6 r_{i}\right)} \text { and }\left|\nabla \eta_{i}\right| \leq 2 / r_{i} .
$$

Then

$$
\begin{aligned}
\gamma_{\boldsymbol{F}}\left(\widehat{B}_{i}\right) & \leq \int_{\mathbb{R}^{n}}\left(\boldsymbol{F}\left(\left|\eta_{i}\right|\right)+\boldsymbol{F}\left(\left|\nabla \eta_{i}\right|\right)\right) d y \leq \int_{B\left(x_{i}, 6 r_{i}\right)}\left(\boldsymbol{F}(1)+\boldsymbol{F}\left(2 / r_{i}\right)\right) d y \\
& \leq C r_{i}^{n} \boldsymbol{F}\left(1 / r_{i}\right) \leq \frac{C}{\varepsilon} \int_{B_{i}} \boldsymbol{F}(|g|) d y .
\end{aligned}
$$

Hence by Theorem 3.5(v),

$$
\begin{aligned}
\gamma_{\boldsymbol{F}}\left(A_{\varepsilon}\right) & \leq \gamma_{\boldsymbol{F}}\left(\bigcup_{i=1}^{\infty} \widehat{B}_{i}\right) \leq \sum_{i=1}^{\infty} \gamma_{\boldsymbol{F}}\left(\widehat{B}_{i}\right) \leq \frac{C}{\varepsilon} \sum_{i=1}^{\infty} \int_{B_{i}} \boldsymbol{F}(|g|) d y \\
& \leq \frac{C}{\varepsilon} \int_{G} \boldsymbol{F}(|g|) d y .
\end{aligned}
$$

Now, the right hand side of (5.3) can be made arbitrarily small with an appropriate choice of $G$ and hence $\gamma_{\boldsymbol{F}}\left(A_{\varepsilon}\right)=0$. Again invoking Theorem $3.5(\mathrm{v})$, we get $\gamma_{\boldsymbol{F}}\left(\bigcup_{\varepsilon>0} A_{\varepsilon}\right)=0$, which concludes the proof.

Cianchi $[\mathrm{C} 1]$ proved the following embedding theorem:

Proposition 5.2. Let $n \geq 2$ and $\boldsymbol{F}$ be a Young function such that

$$
\int_{0}^{T}\left(\frac{t}{\boldsymbol{F}(t)}\right)^{n^{\prime}-1} d t<\infty \text { and } \int_{T}^{\infty}\left(\frac{t}{\boldsymbol{F}(t)}\right)^{n^{\prime}-1} d t=\infty
$$

for all $T>0$, where $n^{\prime}$ is the Hölder conjugate of $n$. Let $\boldsymbol{F}_{n}$ be defined by

$$
\boldsymbol{F}_{n}\left(\left(\int_{0}^{T}\left(\frac{t}{\boldsymbol{F}(t)}\right)^{n^{\prime}-1} d t\right)^{1 / n^{\prime}}\right)=\boldsymbol{F}(T), \quad T>0 .
$$

Then for each $u \in W^{1, \boldsymbol{F}}\left(\mathbb{R}^{n}\right)$ we have

$$
\int_{\mathbb{R}^{n}} \boldsymbol{F}_{n}\left(\frac{|u(y)|}{8 \boldsymbol{\alpha}(n)^{-1 / n} I^{1 / n}}\right) d y \leq I, \quad \text { where } \quad I=\int_{\mathbb{R}^{n}} \boldsymbol{F}(|\nabla u|) d x .
$$

Proposition 5.3. Let $B=B(z, r)$. Let $n \geq 2$ and let $\boldsymbol{F}$ be a Young function satisfying global $\Delta_{2}$ and (5.4). Let $\boldsymbol{F}_{n}$ be as in Proposition 5.2. If $u \in W^{1, \boldsymbol{F}}(B)$ and

$$
I=\int_{B} \boldsymbol{F}(|\nabla u|) d x
$$

then there is a constant $C=C(n)$ such that

$$
\int_{B} \boldsymbol{F}_{n}\left(\frac{\left|u-\bar{u}_{B}\right|}{C I^{1 / n}}\right) d y \leq C I .
$$


Proof. We may assume that $\bar{u}_{B}=0$. By Proposition 2.12, there is $v \in$ $W^{1, \boldsymbol{F}}\left(\mathbb{R}^{n}\right)$ such that $v=u$ on $B$ and

$$
\int_{\mathbb{R}^{n}} \boldsymbol{F}(|\nabla v|) d x \leq C \int_{B}(\boldsymbol{F}(|\nabla u|)+\boldsymbol{F}(|u| / r)) d x .
$$

Using Proposition 2.14 now gives

$$
\int_{\mathbb{R}^{n}} \boldsymbol{F}(|\nabla v|) d x \leq C \int_{B} \boldsymbol{F}(|\nabla u|) d x \leq C I
$$

and thus

$$
\int_{B} \boldsymbol{F}_{n}\left(\frac{|u(y)|}{C I^{1 / n}}\right) d y \leq \int_{\mathbb{R}^{n}} \boldsymbol{F}_{n}\left(\frac{|v(y)|}{C I^{1 / n}}\right) d y \leq C I .
$$

COROLlary 5.4. Let $\boldsymbol{F}$ be a Young function satisfying global $\Delta_{2}$ and (5.4), and let $\boldsymbol{F}_{n}$ be as in Proposition 5.2. Suppose that $u \in W_{\mathrm{loc}}^{1, \boldsymbol{F}}\left(\mathbb{R}^{n}\right)$. Then

$$
\lim _{r \rightarrow 0+} \frac{1}{\boldsymbol{F}(1 / r)} f_{B(z, r)} \boldsymbol{F}_{n}\left(\frac{|u(x)-\bar{u}(z, r)|}{r \boldsymbol{F}(1 / r)^{1 / n}}\right) d x=0
$$

for $\gamma_{\boldsymbol{F}^{-}}$..e. $z \in \mathbb{R}^{n}$.

Proof. Let $z \in \mathbb{R}^{n}$ be a Lebesgue point for $u$ satisfying (5.2). With $g=|\nabla u|$ in Lemma 5.1, we know that $\gamma_{\boldsymbol{F}}$-q.e. $z \in \mathbb{R}^{n}$ has this property. Hence

$$
\int_{B(z, r)} \boldsymbol{F}(|\nabla u|) d x=\omega(r) r^{n} \boldsymbol{F}(1 / r), \quad \text { where } \quad \lim _{r \rightarrow 0+} \omega(r)=0 .
$$

By Proposition 5.3, it follows that

$$
\int_{B} \boldsymbol{F}_{n}\left(\frac{|u(x)-\bar{u}(z, r)|}{C \omega(r)^{1 / n} r \boldsymbol{F}(1 / r)^{1 / n}}\right) d x \leq C \omega(r) r^{n} \boldsymbol{F}(1 / r) .
$$

This proves (5.5).

REMARK 5.5. Corollary 5.4 is not precisely saying what we want because we subtract $\bar{u}(x, r)$ and not $\bar{u}(x)$. In Theorem 5.8 below we present a version which really generalizes (5.1), but at the expense of an additional restriction on $\boldsymbol{F}$ which is described next.

Definition 5.6. Given a global $\Delta_{2}$ Young function $\boldsymbol{F}$ for which $t \mapsto$ $t / \boldsymbol{F}(t)^{1 / n}$ is injective we define $\boldsymbol{F}_{*}$ by

$$
\boldsymbol{F}_{*}\left(\frac{t}{\boldsymbol{F}(t)^{1 / n}}\right)=\boldsymbol{F}(t) .
$$

LemMA 5.7. Let $\boldsymbol{F}$ be a Young function such that $t \mapsto t^{-p} \boldsymbol{F}(t)$ is nonincreasing for some $p<n$. Then $\boldsymbol{F}$ satisfies (5.4), $\boldsymbol{F}_{*}$ is nondecreasing and 
doubling and

$$
\boldsymbol{F}_{*}(s) \leq C \boldsymbol{F}_{n}(s), \quad s>0 .
$$

Proof. We have

$$
\begin{aligned}
\int_{0}^{T}\left(\frac{t}{\boldsymbol{F}(t)}\right)^{n^{\prime}-1} d t & =\int_{0}^{T} t^{-\frac{p-1}{n-1}}\left(\frac{t^{p}}{\boldsymbol{F}(t)}\right)^{n^{\prime}-1} d t \\
& \leq\left(\frac{T^{p}}{\boldsymbol{F}(T)}\right)^{n^{\prime}-1} \int_{0}^{T} t^{-\frac{p-1}{n-1}} d t=\frac{T^{n^{\prime}}}{\boldsymbol{F}(T)^{n^{\prime}-1}}<\infty
\end{aligned}
$$

and

$$
\begin{aligned}
\int_{T}^{\infty}\left(\frac{t}{\boldsymbol{F}(t)}\right)^{n^{\prime}-1} d t & =\int_{T}^{\infty} t^{-\frac{p-1}{n-1}}\left(\frac{t^{p}}{\boldsymbol{F}(t)}\right)^{n^{\prime}-1} d t \\
& \geq\left(\frac{T^{p}}{\boldsymbol{F}(T)}\right)^{n^{\prime}-1} \int_{T}^{\infty} t^{-\frac{p-1}{n-1}} d t=\infty,
\end{aligned}
$$

so $\boldsymbol{F}$ satisfies (5.4). For $s>t$ we have

$$
t^{-n} \boldsymbol{F}(t) \geq t^{p-n} t^{-p} \boldsymbol{F}(t) \geq t^{p-n} s^{-p} \boldsymbol{F}(s) \geq\left(\frac{s}{t}\right)^{n-p} s^{-n} \boldsymbol{F}(s),
$$

which, with $\lambda=2^{n /(n-p)}$, implies that

$$
\frac{\boldsymbol{F}(\lambda t)}{(\lambda t)^{n}} \leq \frac{\boldsymbol{F}(t)}{(2 t)^{n}} .
$$

Therefore

$$
\boldsymbol{F}_{*}\left(\frac{2 t}{\boldsymbol{F}(t)^{1 / n}}\right) \leq \boldsymbol{F}_{*}\left(\frac{\lambda t}{\boldsymbol{F}(\lambda t)^{1 / n}}\right)=\boldsymbol{F}(\lambda t) \leq \lambda^{p} \boldsymbol{F}(t)=\lambda^{p} \boldsymbol{F}_{*}\left(\frac{t}{\boldsymbol{F}(t)^{1 / n}}\right),
$$

so that $\boldsymbol{F}_{*}$ is doubling. It follows that

$$
\begin{aligned}
\boldsymbol{F}_{*}\left(\left(\int_{0}^{T}\left(\frac{t}{\boldsymbol{F}(t)}\right)^{n^{\prime}-1} d t\right)^{1 / n^{\prime}}\right) & \leq \boldsymbol{F}_{*}\left(C \frac{T}{\boldsymbol{F}(T)^{1 / n}}\right) \\
& \leq C \boldsymbol{F}_{*}\left(\frac{T}{\boldsymbol{F}(T)^{1 / n}}\right)=C \boldsymbol{F}(T) \\
& \leq C \boldsymbol{F}_{n}\left(\left(\int_{0}^{T}\left(\frac{t}{\boldsymbol{F}(t)}\right)^{n^{\prime}-1} d t\right)^{1 / n^{\prime}}\right),
\end{aligned}
$$

so we arrive at (5.6).

Due to the monotonicity and doubling property of $\boldsymbol{F}_{*}$, we observe

$$
\boldsymbol{F}_{*}(s+t) \leq C\left(\boldsymbol{F}_{*}(s)+\boldsymbol{F}_{*}(t)\right), \quad s, t \geq 0,
$$

which allows us to handle the difference between $\bar{u}(x)$ and $\bar{u}(x, r)$. 
THeOREM 5.8. Let $\boldsymbol{F}$ be a Young function satisfying $\Delta_{2}$ globally. Suppose that $u \in W_{\text {loc }}^{1, \boldsymbol{F}}\left(\mathbb{R}^{n}\right)$ is precisely represented and that $t \mapsto t^{-p} \boldsymbol{F}(t)$ is nonincreasing, $p<n$. Then

$$
\lim _{r \rightarrow 0+} \frac{1}{\boldsymbol{F}(1 / r)} \underset{B(z, r)}{f} \boldsymbol{F}_{*}\left(\frac{|u(x)-\bar{u}(z)|}{r \boldsymbol{F}(1 / r)^{1 / n}}\right) d x=0
$$

for $\gamma_{\boldsymbol{F}^{-}}$q.e. $z \in \mathbb{R}^{n}$.

Proof. Let $z \in \mathbb{R}^{n}$ be a Lebesgue point for $u$ satisfying (5.5). By Corollary 5.4, we know that $\gamma_{\boldsymbol{F}}$-q.e. $z \in \mathbb{R}^{n}$ has this property. Then, by Lemma 5.7 ,

$$
\lim _{r \rightarrow 0+} \frac{1}{\boldsymbol{F}(1 / r)} \int_{B(z, r)} \boldsymbol{F}_{*}\left(\frac{|u(x)-\bar{u}(z, r)|}{r \boldsymbol{F}(1 / r)^{1 / n}}\right) d x=0 .
$$

By (5.7), we have

$$
\begin{aligned}
& f_{B(z, r)} \boldsymbol{F}_{*}\left(\frac{|u(x)-\bar{u}(z)|}{r \boldsymbol{F}(1 / r)^{1 / n}}\right) d x \\
\leq & C \operatorname{f(z,r)}_{*} \boldsymbol{F}_{*}\left(\frac{|u(x)-\bar{u}(z, r)|}{r \boldsymbol{F}(1 / r)^{1 / n}}\right) d x+C{\underset{B(z, r)}{f} \boldsymbol{F}_{*}\left(\frac{|\bar{u}(z, r)-\bar{u}(z)|}{r \boldsymbol{F}(1 / r)^{1 / n}}\right) d x .}^{f} .
\end{aligned}
$$

Since

$$
o(z, r):=\bar{u}(z, r)-\bar{u}(z) \rightarrow 0 \quad \text { as } r \rightarrow 0^{+},
$$

we estimate

$$
\begin{aligned}
& \boldsymbol{F}_{*}\left(\frac{|\bar{u}(x, r)-\bar{u}(z)|}{r \boldsymbol{F}(1 / r)^{1 / n}}\right) \\
= & \boldsymbol{F}_{*}\left(\frac{o(z, r)}{r \boldsymbol{F}(1 / r)^{1 / n}}\right)=C \boldsymbol{F}_{*}\left(\frac{o(z, r) r^{-1}}{\boldsymbol{F}\left(o(z, r) r^{-1}\right)^{1 / n}}\right)=C \boldsymbol{F}(o(z, r)(1 / r))
\end{aligned}
$$

for all $r$ satisfying $o(z, r)<1$. Combining (5.10), (5.9) and (5.11) we obtain the assertion.

REMARK 5.9. If we use the notation

$$
\boldsymbol{F}_{*}(t, r)=\frac{\boldsymbol{F}_{*}(t)}{r \boldsymbol{F}(1 / r)^{1 / n}},
$$

we can write (5.8) in the "symmetric form"

$$
\lim _{r \rightarrow 0+} f_{B(z, r)} \frac{\boldsymbol{F}_{*}(|u(x)-\bar{u}(z)|, r)}{\boldsymbol{F}_{*}(1, r)} d x=0 .
$$

6. Capacitary distribution. In this auxiliary section we shall mention some elementary properties of capacitary extremals. 
Definition 6.1. We suppose that $\Omega \subset \mathbb{R}^{n}$ is a Lipschitz domain with finite Lebesgue measure and that $\boldsymbol{F}$ is a Young function satisfying $\Delta_{2}$ globally. We define the capacity $\mathrm{c}_{\boldsymbol{F}}(\cdot, \Omega)$ as a particular case of the general capacity (3.1) from Definition 3.2, obtained by setting $c=0$.

In light of Proposition 2.13, the continuity assumption of Definition 3.2 is satisfied and

$$
\mathcal{U}=W_{0}^{1, \boldsymbol{F}}(\Omega) .
$$

In view of the good behavior of $\boldsymbol{F}$ with respect to truncation the capacity $\mathrm{c}_{\boldsymbol{F}}(\cdot, \Omega)$ is given by

$$
\mathrm{c}_{\boldsymbol{F}}(E, \Omega)=\inf \left\{\int_{\Omega} \boldsymbol{F}(|\nabla u|) d x: u \in W_{0}^{1, \boldsymbol{F}}(\Omega), E \subset \operatorname{int}\{u \geq 1\}\right\} .
$$

We use the Lipschitz boundary of $\Omega$ to observe that

$$
W_{0}^{1, \boldsymbol{F}}(\Omega)=W^{1, \boldsymbol{F}}(\Omega) \cap W_{0}^{1,1}(\Omega)
$$

because the "zero trace" means for both spaces simply that the zero extension is in $W_{\text {loc }}^{1,1}\left(\mathbb{R}^{n}\right)$. This enables us to verify the compactness assumption of Definition 3.2 similarly to the case of $\gamma_{\boldsymbol{F}}$ (cf. paragraph after Definition 4.1). If the space $W_{0}^{1, \boldsymbol{F}}(\Omega)$ is reflexive, we do not need to assume that $\Omega$ is Lipschitz and the assumption $|\Omega|<\infty$ is enough.

Anticipating the approximation in Section 7 below, we will assume that $\boldsymbol{F}$ is a continuously differentiable and strictly convex $N$-function. We define

$$
\mathbf{A}(\xi)=\frac{\boldsymbol{F}^{\prime}(|\xi|)}{|\xi|} \xi
$$

Proposition 6.2. Suppose that $G \subset \subset \Omega$ is an open set and let

$$
\mathcal{K}(G)=W_{0}^{1, \boldsymbol{F}}(\Omega) \cap\{u \geq 1 \text { on } G\} .
$$

Then there is a unique capacitary extremal $u_{G} \in \mathcal{K}(G)$ for $\mathrm{c}_{\boldsymbol{F}}(G, \Omega)$. Moreover, there is a finite Radon measure $\mu_{G}$ on $\Omega$ such that

$$
\int_{\Omega} \mathbf{A}\left(\nabla u_{G}\right) \nabla \varphi d x=\int_{\Omega} \varphi d \mu_{G}
$$

for each $\varphi=\mathcal{D}(\Omega)$. If $v \in \mathcal{K}(G)$, then

$$
\int_{\Omega} \mathbf{A}\left(\nabla u_{G}\right) \cdot\left(\nabla v-\nabla u_{G}\right) d x \geq 0 .
$$

Proof. For the existence and uniqueness of $u_{G}$ see Proposition 3.6 and Remark 3.7. If $v \in \mathcal{K}(G)$ and $t>0$ then $u_{G}+t\left(v-u_{G}\right) \in \mathcal{K}(G)$, hence

$$
\int_{\Omega} \boldsymbol{F}\left(\left|\nabla\left(u_{G}+t\left(v-u_{G}\right)\right)\right|\right) d x \geq \int_{\Omega} \boldsymbol{F}\left(\left|\nabla u_{G}\right|\right) d x .
$$


The variational inequality (6.2) is obtained by the differentiation of

$$
t \mapsto \int_{\Omega} \boldsymbol{F}\left(\left|\nabla\left(u_{G}+t\left(v-u_{G}\right)\right)\right|\right) d x
$$

at $t=0+$. Setting $v=u+\varphi$, where $\varphi \in \mathcal{D}(\Omega)$ is nonnegative, we obtain

$$
\int_{\Omega} \mathbf{A}\left(\nabla u_{G}\right) \cdot \nabla \varphi d x \geq 0 \quad \forall \varphi \in \mathcal{D}^{+}(\Omega) .
$$

It follows that

$$
-\operatorname{div} \mathbf{A}\left(\nabla u_{G}\right): \varphi \mapsto \int_{\Omega} \mathbf{A}\left(\nabla u_{G}\right) \cdot \nabla \varphi d x
$$

is a positive distribution on $\mathcal{D}(\Omega)$, and thus we may apply the Riesz representation theorem to deduce the existence of $\mu_{G}$.

Definition 6.3. The measure $\mu_{G}$ from Proposition 6.2 is called the capacitary distribution of $\mathrm{c}_{\boldsymbol{F}}(G, \Omega)$.

Lemma 6.4. Let $G \subset \subset \Omega$ be an open set and $\mu$ be the capacitary distribution of $\mathrm{c}_{\boldsymbol{F}}(G, \Omega)$. Then

$$
\mu(G) \leq p \mathrm{c}_{\boldsymbol{F}}(G, \Omega),
$$

where $p$ is the constant from Proposition 2.6(iv).

Proof. Let $u$ be the capacitary extremal for $\mathrm{c}_{\boldsymbol{F}}(G, \Omega)$ and $\eta \in \mathcal{D}(\Omega), 0 \leq$ $\eta \leq 1$. We use the fact that $2 u-\eta \in \mathcal{K}(G)$ together with (6.2), Proposition 2.6(iii), and the definition of extremal to obtain

$$
\int_{\Omega} \eta d \mu=\int_{\Omega} \mathbf{A}(\nabla u) \cdot \nabla \eta d x \leq \int_{\Omega} \mathbf{A}(\nabla u) \cdot \nabla u d x \leq p \int_{\Omega} \boldsymbol{F}(|\nabla u|) d x .
$$

Letting $\eta \rightarrow 1$ we reach the conclusion.

7. Smoothing of Young functions. In this section we consider an $N$-function $\boldsymbol{G}$ and a Young function $\boldsymbol{F}$ satisfying

$$
\int_{0}^{\infty}\left[\boldsymbol{G}^{\prime}\right]^{-1}\left(\frac{1}{\boldsymbol{F}^{\prime}(t)}\right) d t<\infty
$$

where $\left[\boldsymbol{G}^{\prime}\right]^{-1}$ is understood as in Definition 2.17.

Lemma 7.1. Suppose that $\boldsymbol{F}$ and $\boldsymbol{G}$ are as above and (7.1) holds. Then there is a Young function $\boldsymbol{G}_{1}$ such that

(i) $\boldsymbol{G}_{1}$ is an $N$-function.

(ii) $\boldsymbol{G}_{1} \leq \boldsymbol{G}$.

(iii) $\int_{0}^{\infty}\left[\boldsymbol{G}_{1}^{\prime}\right]^{-1}\left(1 / \boldsymbol{F}^{\prime}(t)\right) d t \leq C \int_{0}^{\infty}\left[\boldsymbol{G}^{\prime}\right]^{-1}\left(1 / \boldsymbol{F}^{\prime}(t)\right) d t$.

(iv) If $\boldsymbol{G}$ satisfies $\Delta_{2}$ globally, then so does $\boldsymbol{G}_{1}$. If $\boldsymbol{G}$ satisfies $\nabla_{2}$ globally, then so does $\boldsymbol{G}_{1}$. 
(v) $G_{1}^{\prime}$ is continuous.

(vi) $G_{1}^{\prime}$ is strictly increasing.

Proof. Let $g=\left[\boldsymbol{G}^{\prime}\right]^{-1}$. First we construct a nondecreasing continuous function $\widetilde{g}$ such that

$$
g(s) \leq \widetilde{g}(s) \leq g(2 s)
$$

and

$$
\int_{0}^{\infty} \widetilde{g}\left(\frac{1}{\boldsymbol{F}^{\prime}(t)}\right) d t \leq 2 \int_{0}^{\infty} g\left(\frac{1}{\boldsymbol{F}^{\prime}(t)}\right) d t .
$$

For each $j \in \mathbb{Z}$ we define $g_{j}$ on $[0, \infty)$ so that on each interval

$$
I_{i, j}:=\left[(i-1) 2^{-j}, i 2^{-j}\right], \quad i \in \mathbb{N},
$$

$g_{j}$ is the upper concave envelope of $g$ with respect to $I_{i, j}$. Since $g$ is nondecreasing and right continuous, each function $g_{j}$ is nondecreasing, continuous, and satisfies $g_{j}=g$ at the endpoints of each interval $I_{i, j}$. For fixed $k \in \mathbb{Z}$, $g_{j}$ converges to $g$ on $\left[2^{-k}, 2^{-k+1}\right]$. Hence there exists $j(k) \geq k$ such that

$$
\int_{E_{k}} g_{j(k)}\left(\frac{1}{\boldsymbol{F}_{1}^{\prime}(t)}\right) d t \leq 2 \int_{E_{k}} g\left(\frac{1}{\boldsymbol{F}^{\prime}(t)}\right) d t
$$

where

$$
E_{k}:=\left\{t \in(0, \infty): 2^{-k} \leq 1 / \boldsymbol{F}_{1}^{\prime}(t)<2^{-k+1}\right\}
$$

We define

$$
\widetilde{g}(s)=g_{j(k)}(s), \quad s \in\left[2^{-k}, 2^{-k+1}\right] .
$$

Since $j(k) \geq k$, we have $g_{j(k)}(s)=g(s)$ at the endpoints $s=2^{-k}$ and $s=2^{-k+1}$, so the definition is not ambiguous at the breakpoints. This coincidence also implies (7.2). It is easily seen from (7.2) that if $g$ is doubling, then so is $\widetilde{g}$, and if $g^{-1}$ is doubling, then so is $\widetilde{g}^{-1}$.

Obviously, $g$ has all properties that we require from $\left[\boldsymbol{G}_{1}^{\prime}\right]^{-1}$ except for strict monotonicity. As the next step, we find points $a_{k}, k \in \mathbb{Z}$, so that

$$
a_{k+1}>a_{k}, \quad \lim _{k \rightarrow-\infty} a_{k}=0, \quad \lim _{k \rightarrow \infty} a_{k}=\infty, \quad \widetilde{g}\left(a_{k}\right)<\widetilde{g}\left(a_{k+1}\right) .
$$

We define a piecewise linear function $h$ such that $h$ is linear on each interval $\left[a_{k}, a_{k+1}\right]$ and

$$
h\left(a_{k+1}\right)=\widetilde{g}\left(a_{k}\right) .
$$

Then $h$ is continuous, strictly increasing and $0 \leq h \leq \widetilde{g}$. Clearly, if $\boldsymbol{G}_{1}$ is a Young function such that

$$
\left[\boldsymbol{G}_{1}^{\prime}\right]^{-1}=\widetilde{g}+h,
$$

then $\boldsymbol{G}_{1}$ has all the required properties. 
Lemma 7.1 shows that there is no loss of generality in our consideration of (7.1) if we suppose that the derivative of $\boldsymbol{G}$ is continuous and strictly increasing. We will show that $\boldsymbol{F}$ may be replaced by an $N$-function $\boldsymbol{F}_{1} \leq \boldsymbol{F}$ which inherits good properties of $\boldsymbol{G}$ and preserves (7.1). Consider an exponent $0<\alpha<1$. Define

$$
\mathbf{k}(s)=(1+\alpha) \min \left\{s^{\alpha}, s^{-\alpha}\right\} .
$$

The modified Young function $\boldsymbol{F}_{1}$ will be defined by $\boldsymbol{F}_{1}(0)=0$ and

$$
\frac{1}{\boldsymbol{F}_{1}^{\prime}(t)}=\boldsymbol{G}^{\prime}\left(\int_{0}^{\infty} \mathbf{k}\left(\frac{\lambda}{t}\right)\left[\boldsymbol{G}^{\prime}\right]^{-1}\left(\frac{1}{\boldsymbol{F}^{\prime}(\lambda)}\right) \frac{d \lambda}{t}\right), \quad t>0 .
$$

Theorem 7.2. Let $\boldsymbol{F}$ and $\boldsymbol{G}$ be given as in (7.1), with $\boldsymbol{G}^{\prime}$ strictly increasing and continuous, and let $\boldsymbol{F}_{1}$ be defined by (7.3). Then:

(i) $\boldsymbol{F}_{1}$ is an $N$-function.

(ii) $\boldsymbol{F}_{1} \leq \boldsymbol{F}$.

(iii) $\int_{0}^{\infty}\left[\boldsymbol{G}^{\prime}\right]^{-1}\left(1 / \boldsymbol{F}_{1}^{\prime}(t)\right) d t \leq C \int_{0}^{\infty}\left[\boldsymbol{G}^{\prime}\right]^{-1}\left(1 / \boldsymbol{F}^{\prime}(t)\right) d t$ with $C=C(\alpha)$.

(iv) If $\boldsymbol{G}$ is $\Delta_{2}$, then so is $\boldsymbol{F}_{1}$. If $\boldsymbol{G}$ is $\nabla_{2}$, then so is $\boldsymbol{F}_{1}$.

(v) $\boldsymbol{F}_{1}^{\prime}$ is continuous.

(vi) $\boldsymbol{F}_{1}^{\prime}$ is strictly increasing.

Proof. (i), (v), (vi). Observe that the function

$$
t \mapsto \int_{0}^{\infty} \mathbf{k}\left(\frac{\lambda}{t}\right)\left[\boldsymbol{G}^{\prime}\right]^{-1}\left(\frac{1}{\boldsymbol{F}^{\prime}(\lambda)}\right) \frac{d \lambda}{t}
$$

is strictly decreasing and continuous, which implies (v) and (vi). Since

$$
\frac{1}{\boldsymbol{F}_{1}^{\prime}(t)} \geq \boldsymbol{G}^{\prime}\left(t^{\alpha-1} \int_{t}^{\infty} \lambda^{-\alpha}\left[\boldsymbol{G}^{\prime}\right]^{-1}\left(\frac{1}{\boldsymbol{F}^{\prime}(\lambda)}\right) d \lambda\right)
$$

we have

$$
\frac{1}{\boldsymbol{F}_{1}^{\prime}(t)} \rightarrow \infty \quad \text { as } t \rightarrow 0 .
$$

Similarly, since $\mathbf{k}(\lambda / t) \leq 1+\alpha$, we have

$$
\frac{1}{\boldsymbol{F}_{1}^{\prime}(t)} \leq \boldsymbol{G}^{\prime}\left(\frac{1+\alpha}{t} \int_{0}^{\infty}\left[\boldsymbol{G}^{\prime}\right]^{-1}\left(\frac{1}{\boldsymbol{F}^{\prime}(\lambda)}\right) d \lambda\right),
$$

so it follows that

$$
\frac{1}{\boldsymbol{F}_{1}^{\prime}(t)} \rightarrow 0 \quad \text { as } t \rightarrow \infty
$$


(ii) Since $\boldsymbol{F}^{\prime}(\lambda) \leq \boldsymbol{F}^{\prime}(t)$ for $0<\lambda<t$, we have

$$
\begin{aligned}
\frac{1}{\boldsymbol{F}_{1}^{\prime}(t)} & \geq \boldsymbol{G}^{\prime}\left(\int_{0}^{t} \mathbf{k}\left(\frac{\lambda}{t}\right)\left[\boldsymbol{G}^{\prime}\right]^{-1}\left(\frac{1}{\boldsymbol{F}^{\prime}(\lambda)}\right) \frac{d \lambda}{t}\right) \\
& \geq \boldsymbol{G}^{\prime}\left(\left[\boldsymbol{G}^{\prime}\right]^{-1}\left(\frac{1}{\boldsymbol{F}^{\prime}(t)}\right) \int_{0}^{t} \mathbf{k}\left(\frac{\lambda}{t}\right) \frac{d \lambda}{t}\right) \\
& =\boldsymbol{G}^{\prime}\left(\left[\boldsymbol{G}^{\prime}\right]^{-1}\left(\frac{1}{\boldsymbol{F}^{\prime}(t)}\right)\right) \geq \frac{1}{\boldsymbol{F}^{\prime}(t)}
\end{aligned}
$$

and thus $\boldsymbol{F}_{1} \leq \boldsymbol{F}$.

(iii) We have

$$
\begin{aligned}
\int_{0}^{\infty}\left[\boldsymbol{G}^{\prime}\right]^{-1}\left(\frac{1}{\boldsymbol{F}_{1}^{\prime}(t)}\right) d t & =\int_{0}^{\infty}\left(\int_{0}^{\infty} \mathbf{k}\left(\frac{\lambda}{t}\right)\left[\boldsymbol{G}^{\prime}\right]^{-1}\left(\frac{1}{\boldsymbol{F}^{\prime}(\lambda)}\right) \frac{d \lambda}{t}\right) d t \\
& =\int_{0}^{\infty}\left(\int_{0}^{\infty} \mathbf{k}\left(\frac{\lambda}{t}\right)\left[\boldsymbol{G}^{\prime}\right]^{-1}\left(\frac{1}{\boldsymbol{F}^{\prime}(\lambda)}\right) \frac{d t}{t}\right) d \lambda \\
& \leq \int_{0}^{\infty}\left(\int_{0}^{\infty} \mathbf{k}(s) \frac{d s}{s}\right)\left[\boldsymbol{G}^{\prime}\right]^{-1}\left(\frac{1}{\boldsymbol{F}^{\prime}(\lambda)}\right) d \lambda \\
& =\frac{2}{\alpha}(\alpha+1) \int_{0}^{\infty}\left[\boldsymbol{G}^{\prime}\right]^{-1}\left(\frac{1}{\boldsymbol{F}^{\prime}(\lambda)}\right) d \lambda .
\end{aligned}
$$

(iv) Observe that $s^{\alpha} \mathbf{k}(s)$ is nondecreasing and $s^{-\alpha} \mathbf{k}(s)$ is nonincreasing. Thus for $0<s<t$ we have

$$
\mathbf{k}\left(\frac{\lambda}{s}\right) \frac{1}{s} \geq\left(\frac{s}{t}\right)^{\alpha-1} \mathbf{k}\left(\frac{\lambda}{t}\right) \frac{1}{t}, \quad \mathbf{k}\left(\frac{\lambda}{s}\right) \frac{1}{s} \leq\left(\frac{s}{t}\right)^{-\alpha-1} \mathbf{k}\left(\frac{\lambda}{t}\right) \frac{1}{t} .
$$

Hence

$$
\begin{aligned}
& {\left[\boldsymbol{G}^{\prime}\right]^{-1}\left(\frac{1}{\boldsymbol{F}_{1}^{\prime}(s)}\right) \geq\left(\frac{s}{t}\right)^{\alpha-1}\left[\boldsymbol{G}^{\prime}\right]^{-1}\left(\frac{1}{\boldsymbol{F}_{1}^{\prime}(t)}\right),} \\
& {\left[\boldsymbol{G}^{\prime}\right]^{-1}\left(\frac{1}{\boldsymbol{F}_{1}^{\prime}(s)}\right) \leq\left(\frac{s}{t}\right)^{-\alpha-1}\left[\boldsymbol{G}^{\prime}\right]^{-1}\left(\frac{1}{\boldsymbol{F}_{1}^{\prime}(t)}\right)}
\end{aligned}
$$

since $\left[\boldsymbol{G}^{\prime}\right]^{-1}$ is nondecreasing.

Suppose that $\boldsymbol{G}$ satisfies $\Delta_{2}$ globally. By Proposition 2.6 there is a constant $K>1$ such that

$$
\boldsymbol{G}^{\prime}(2 t) \leq K \boldsymbol{G}^{\prime}(t) \text { for all } t>0 .
$$

Setting $t=2^{1 /(\alpha+1)} s$ we have

$$
\left[\boldsymbol{G}^{\prime}\right]^{-1}\left(\frac{1}{\boldsymbol{F}_{1}^{\prime}(s)}\right) \leq\left(\frac{s}{t}\right)^{-1-\alpha}\left[\boldsymbol{G}^{\prime}\right]^{-1}\left(\frac{1}{\boldsymbol{F}_{1}^{\prime}(t)}\right)=2\left[\boldsymbol{G}^{\prime}\right]^{-1}\left(\frac{1}{\boldsymbol{F}_{1}^{\prime}(t)}\right),
$$


so the monotonicity of $\boldsymbol{G}^{\prime}$ implies

$$
\frac{1}{\boldsymbol{F}_{1}^{\prime}(s)} \leq \boldsymbol{G}^{\prime}\left(2\left[\boldsymbol{G}^{\prime}\right]^{-1}\left(\frac{1}{\boldsymbol{F}_{1}^{\prime}(t)}\right)\right) \leq K \boldsymbol{G}^{\prime}\left(\left[\boldsymbol{G}^{\prime}\right]^{-1}\left(\frac{1}{\boldsymbol{F}_{1}^{\prime}(t)}\right)\right)=K \frac{1}{\boldsymbol{F}_{1}^{\prime}(t)},
$$

that is,

$$
\boldsymbol{F}_{1}^{\prime}\left(2^{1 /(\alpha+1)} s\right) \leq K \boldsymbol{F}_{1}^{\prime}(s)
$$

for all $s>0$. This implies that $\boldsymbol{F}_{1}$ satisfies $\Delta_{2}$ globally.

Suppose now that $\boldsymbol{G}$ satisfies $\nabla_{2}$ globally. Again by Proposition 2.6 there is a constant $K>1$ such that

$$
2 G^{\prime}(t) \leq G^{\prime}(K t) \quad \text { for all } t>0 .
$$

Setting $t=K^{1 /(1-\alpha)} s$ we have

$$
\left[\boldsymbol{G}^{\prime}\right]^{-1}\left(\frac{1}{\boldsymbol{F}_{1}^{\prime}(s)}\right) \geq\left(\frac{s}{t}\right)^{\alpha-1}\left[\boldsymbol{G}^{\prime}\right]^{-1}\left(\frac{1}{\boldsymbol{F}_{1}^{\prime}(t)}\right)=K\left[\boldsymbol{G}^{\prime}\right]^{-1}\left(\frac{1}{\boldsymbol{F}_{1}^{\prime}(t)}\right),
$$

so the monotonicity of $\boldsymbol{G}^{\prime}$ implies

$$
\frac{1}{\boldsymbol{F}_{1}^{\prime}(s)} \geq \boldsymbol{G}^{\prime}\left(K\left[\boldsymbol{G}^{\prime}\right]^{-1}\left(\frac{1}{\boldsymbol{F}_{1}^{\prime}(t)}\right)\right) \geq 2 \boldsymbol{G}^{\prime}\left(\left[\boldsymbol{G}^{\prime}\right]^{-1}\left(\frac{1}{\boldsymbol{F}_{1}^{\prime}(t)}\right)\right) \geq \frac{2}{\boldsymbol{F}_{1}^{\prime}(t)} .
$$

It follows that $2 \boldsymbol{F}_{1}^{\prime}(s) \leq \boldsymbol{F}_{1}^{\prime}\left(2^{1 /(1-\alpha)} s\right)$ for all $s>0$, so $\boldsymbol{F}_{1}$ satisfies $\nabla_{2}$ globally.

LEMma 7.3. Suppose that $\boldsymbol{F}$ satisfies $\Delta_{2}$ globally, $\boldsymbol{F}_{1}$ satisfies $\nabla_{2}$ globally, and $\boldsymbol{F}_{1}^{\prime} \leq \boldsymbol{F}^{\prime}$. Then there exists $\boldsymbol{F}_{2}$ satisfying both $\Delta_{2}$ and $\nabla_{2}$ globally such that

$$
\boldsymbol{F}_{1}^{\prime} \leq \boldsymbol{F}_{2}^{\prime} \leq \boldsymbol{F}_{1}^{\prime}
$$

Proof. Define $f=\boldsymbol{F}^{\prime}, g=\boldsymbol{F}_{1}^{\prime}$. We set

$$
\boldsymbol{F}_{2}(t)=\int_{0}^{t} \phi(s) d s
$$

where $\phi$ is to be constructed. Let $a, b>1$ be constants as in Proposition 2.6 satisfying

$$
f(2 t) \leq a f(t) \quad \text { and } \quad g(b t) \geq 2 g(t)
$$

for $t>0$. Define

$$
\phi(t)=\sup \left\{g(t), \frac{g(2 t)}{a}, \frac{g(4 t)}{a^{2}}, \frac{g(8 t)}{a^{3}}, \ldots\right\} .
$$

We observe that $\phi$ is nondecreasing (as the supremum of a family of nondecreasing functions), and that $\phi \geq g$. On the other hand, the sequence of inequalities

$$
g(t) \leq f(t), \quad \frac{g(2 t)}{a} \leq \frac{f(2 t)}{a} \leq f(t), \quad \frac{g(4 t)}{a^{2}} \leq \frac{f(4 t)}{a^{2}} \leq \frac{f(2 t)}{a} \leq f(t), \quad \ldots
$$


implies that $\phi \leq f$. Now,

$$
\begin{aligned}
\phi(b t) & =\sup \left\{g(b t), \frac{g(2 b t)}{a}, \frac{g(4 b t)}{a^{2}}, \ldots\right\} \\
& \geq \sup \left\{2 g(t), \frac{2 g(2 t)}{a}, \frac{2 g(4 t)}{a^{2}}, \ldots\right\} \geq 2 \phi(t)
\end{aligned}
$$

and

$$
\begin{aligned}
\phi(2 t) & =\sup \left\{g(2 t), \frac{g(4 t)}{a}, \frac{g(8 t)}{a^{2}}, \ldots\right\} \\
& \leq a \sup \left\{\frac{g(2 t)}{a}, \frac{g(4 t)}{a^{2}}, \frac{g(8 t)}{a^{3}}, \ldots\right\} \leq a \phi(t),
\end{aligned}
$$

so $\boldsymbol{F}_{2}$ satisfies $\Delta_{2}$ and $\nabla_{2}$.

COROLlary 7.4. Suppose that $\boldsymbol{F}$ or $\boldsymbol{G}$ satisfies $\Delta_{2}$ globally and that $\boldsymbol{F}$ or $\boldsymbol{G}$ satisfies $\nabla_{2}$ globally. Then there is a Young function $\boldsymbol{F}_{2} \leq \boldsymbol{F}$ satisfying both $\Delta_{2}$ and $\nabla_{2}$ globally such that

$$
\int_{0}^{\infty}\left[\boldsymbol{G}^{\prime}\right]^{-1}\left(\frac{1}{\boldsymbol{F}_{2}^{\prime}(t)}\right) d t \leq C \int_{0}^{\infty}\left[\boldsymbol{G}^{\prime}\right]^{-1}\left(\frac{1}{\boldsymbol{F}^{\prime}(t)}\right) d t .
$$

Proof. If $\boldsymbol{F}$ satisfies $\Delta_{2}$ and $\nabla_{2}$ there is nothing to prove, and if $\boldsymbol{G}$ satisfies $\Delta_{2}$ and $\nabla_{2}$ we set $\boldsymbol{F}_{2}=\boldsymbol{F}_{1}$. If $\boldsymbol{F}$ satisfies $\Delta_{2}$ and $\boldsymbol{G}$ satisfies $\nabla_{2}$, then $\boldsymbol{F}_{1}$ satisfies $\nabla_{2}$ and we can use Lemma 7.3 to produce $\boldsymbol{F}_{2}$. Finally, if $\boldsymbol{F}$ satisfies $\nabla_{2}$ and $\boldsymbol{G}$ satisfies $\Delta_{2}$, then we use a dual version of the previous reasoning to construct $\boldsymbol{G}_{2}$ such that $\boldsymbol{G}_{2}^{\prime} \leq \boldsymbol{G}^{\prime}, \boldsymbol{G}_{2}$ satisfies $\Delta_{2}$ and $\nabla_{2}$, and

$$
\int_{0}^{\infty}\left[\boldsymbol{G}_{2}^{\prime}\right]^{-1}\left(\frac{1}{\boldsymbol{F}^{\prime}(t)}\right) d t \leq C \int_{0}^{\infty}\left[\boldsymbol{G}^{\prime}\right]^{-1}\left(\frac{1}{\boldsymbol{F}^{\prime}(t)}\right) d t .
$$

Then we construct $\boldsymbol{F}_{2}$ from $\boldsymbol{F}$ using $\boldsymbol{G}_{2}$ similarly to the way $\boldsymbol{F}_{1}$ was constructed using $\boldsymbol{G}$.

8. Capacity and Hausdorff content. In this section we compare Hausdorff content and capacity. The estimates of $W^{1, p}$-capacity go back to Frostman $[\mathrm{F}]$ for $p=2$, to Reshetnyak [Re] for the case of general $p$, and in sharp form to Maz'ya and Havin [MH]. The extension to Orlicz-Sobolev capacity has been done by Fiorenza and Prignet $[\mathrm{FP}]$ with the use of deep results on equivalence of certain function spaces and capacities. Here we present an alternative development based on estimates of capacitary extremals.

Definition 8.1. A gauge is a nondecreasing function $h:[0, \infty) \rightarrow[0, \infty)$ with $h(0)=0$. The spherical Hausdorff content induced by $h$ is defined for 
all sets $E \subset \mathbb{R}^{n}$ by

$$
\mathcal{H}_{h}(E)=\inf \left\{\sum_{j} h\left(r_{j}\right): E \subset \bigcup_{j} B\left(x_{j}, r_{j}\right)\right\} .
$$

The maximal function induced by $h$ of a Radon measure $\mu$ is defined by

$$
M_{h, R} \mu(x)=\sup _{0<r<R} \frac{\mu(B(x, r))}{h(r)} .
$$

The relation between $\mathcal{H}_{h}$ and $M_{h, R}$ is given by the following lemma of Cartan which is a modification of the Hardy-Littlewood maximal theorem. See also Bagby and Ziemer [BZ, Lemma 3.2].

Proposition 8.2. Suppose that $h$ is a gauge, $\mu$ is a Radon measure on $\mathbb{R}^{n}$, and $R>0$. There is a constant $C=C(n)$ so that

$$
\mathcal{H}_{h}\left(\left\{M_{h, R} \mu>\lambda\right\}\right) \leq C \frac{\mu\left(\mathbb{R}^{n}\right)}{\lambda} \quad \text { for all } \lambda>0 .
$$

Proof. Define $E_{\lambda}=\left\{M_{h, R} \mu>\lambda\right\}$. For every $x \in E_{\lambda}$ choose $0<r(x)<R$ satisfying $\mu(B(x, r(x)))>\lambda h(r(x))$. By the Besicovitch covering theorem there is an integer $C \geq 1$ and a sequence of balls $B_{j}$ of the form $B\left(x_{j}, r\left(x_{j}\right)\right)$ with centers in $E_{\lambda}$ so that

$$
E_{\lambda} \subset \bigcup_{j=1}^{\infty} B_{j} \quad \text { and } \quad \sum_{J} \chi_{B_{j}} \leq C .
$$

Thus

$$
\mathcal{H}_{h}\left(E_{\lambda}\right) \leq \sum_{j} h\left(r\left(x_{j}\right)\right)<\frac{1}{\lambda} \sum_{j} \mu\left(B_{j}\right) \leq \frac{C}{\lambda} \mu\left(\mathbb{R}^{n}\right) .
$$

Definition 8.3. Given an $N$-function $\boldsymbol{F}$, the $\boldsymbol{F}$-Wolff potential of a Radon measure $\mu$ is defined as

$$
W_{\boldsymbol{F}, R} \mu(x)=\int_{0}^{R}\left[\boldsymbol{F}^{\prime}\right]^{-1}\left(\frac{\mu(B(x, r))}{r^{n-1}}\right) d r .
$$

Next we recall the result of [M1].

Proposition 8.4. Let $B=B(z, 1)$ and let $\mu$ be a finite Radon measure on $\mathbb{R}^{n}$. Let $\boldsymbol{F}$ be a continuously differentiable $N$-function satisfying $\Delta_{2}$ and $\nabla_{2}$ globally and let $p, q>1$ be the constants from Proposition 2.6(iii) and (iv). Suppose that $u \in W_{0}^{1, \boldsymbol{F}}(B)$ is a solution of

$$
\int_{B} \mathbf{A}(\nabla u) \cdot \nabla \varphi d x=\int_{B} \varphi d \mu \quad \text { for each } \varphi \in \mathcal{D}(\Omega),
$$

where $\mathbf{A}$ is given by (6.1). Then there is a constant $C$ depending only on $n$, $p$, and $q$ such that

$$
u(x) \leq C W_{\boldsymbol{F}, 4} \mu(x) \quad \text { for each Lebesgue point } x \text { of } u .
$$


Next we consider a strictly convex continuously differentiable $N$-function $\boldsymbol{F}$ satisfying $\Delta_{2}$ and $\nabla_{2}$ globally such that

$$
\int_{0}^{R}\left[\boldsymbol{F}^{\prime}\right]^{-1}\left(\frac{h(r)}{r^{n-1}}\right) d r<\infty, \quad R>0 .
$$

Lemma 8.5. Assume (8.1) holds. For every $\varepsilon>0$ there exists $\delta>0$ (depending on $\boldsymbol{F}, h$ and $\varepsilon$ ) so that

$$
M_{h, R} \mu(x) \leq \delta \Rightarrow W_{\boldsymbol{F}, R} \mu(x)<\varepsilon
$$

for any Radon measure $\mu$ on $\mathbb{R}^{n}$ and all $x \in \mathbb{R}^{n}$.

Proof. Observe that

$$
W_{\boldsymbol{F}, R} \mu(x)=\int_{0}^{R}\left[\boldsymbol{F}^{\prime}\right]^{-1}\left(\frac{\mu(B(x, r))}{r^{n-1}}\right) d r \leq \int_{0}^{R}\left[\boldsymbol{F}^{\prime}\right]^{-1}\left(\frac{M_{h, R} \mu(x) h(r)}{r^{n-1}}\right) d r .
$$

Since $\lim _{t \rightarrow 0}\left[\boldsymbol{F}^{\prime}\right]^{-1}(t)=0$, inequality (8.1) and the dominated convergence theorem imply that

$$
\lim _{\delta \rightarrow 0} \int_{0}^{R}\left[\boldsymbol{F}^{\prime}\right]^{-1}\left(\frac{\delta h(r)}{r^{n-1}}\right) d r=0
$$

Lemma 8.6. Assume (8.1) holds. Let $B=B(z, 1)$ and let $G \subset \subset B$ be an open set. Then

$$
\mathcal{H}_{h}(G) \leq C \mathrm{c}_{\boldsymbol{F}}(G, B),
$$

where $C$ depends on $\boldsymbol{F}, h, n$.

Proof. Let $u$ be a capacitary extremal for $\mathrm{c}_{\boldsymbol{F}}(G, B)$ and let $\mu$ be its corresponding capacitary distribution. Then $u=1$ on $G$, in particular each point of $G$ is a Lebesgue point of $u$. By Proposition 8.4, there exists $\varepsilon>0$ such that

$$
\varepsilon \leq \varepsilon u(x) \leq W_{\boldsymbol{F}, 4} \mu(x) \quad \text { for every } x \in G .
$$

By Lemma 8.5 there exists $\delta>0$ such that $M_{h, 4} \mu(x) \geq \delta$ at all such points $x$. Since $G \subset\left\{x: M_{h, 4} \mu(x) \geq \delta\right\}$, Proposition 8.2 and Lemma 6.4 imply

$$
\mathcal{H}_{h}(G) \leq \frac{C}{\delta} \mu\left(\mathbb{R}^{n}\right) \leq \frac{C}{\delta} \mathrm{c}_{\boldsymbol{F}}(G, B) .
$$

Lemma 8.7. Assume (8.1) holds. Let $B=B(z, 1)$ and $G \subset B(z, 1 / 2)$ be an open set. Let $u \in W^{1, \boldsymbol{F}}(B), u \geq 1$ on $G$. Then

$$
\mathcal{H}_{h}(G) \leq C \int_{B}(\boldsymbol{F}(|\nabla u|)+\boldsymbol{F}(|u|)) d x
$$

where $C$ depends on $\boldsymbol{F}, h$, and $n$.

Proof. Let $\eta \in \mathcal{D}\left(\mathbb{R}^{n}\right)$ be a cut-off function with

$$
\chi_{B(z, 1 / 2)} \leq \eta \leq \chi_{B(z, 1)} \text { and }|\nabla \eta| \leq 3 .
$$


Then $\eta u$ is a competitor for $\mathrm{c}_{\boldsymbol{F}}(G)$ and

$$
\nabla(\eta u) \leq|\nabla u|+3|u| .
$$

By the $\Delta_{2}$ condition of $\boldsymbol{F}$ this implies

$$
\boldsymbol{F}(|\nabla(\eta u)(x)|) \leq C(\boldsymbol{F}(|u(x)|)+\boldsymbol{F}(|\nabla u(x)|))
$$

for all $x \in B$. The assertion follows from Lemma 8.6.

TheOREM 8.8. Assume (8.1) holds. There is a constant $C$ depending only on $\boldsymbol{F}, h$, and $n$ such that

$$
\mathcal{H}_{h}(E) \leq C \gamma_{\boldsymbol{F}}(E) \quad \text { for all } E \subset \mathbb{R}^{n} .
$$

Proof. Let $G \supset E$ be open and let $u$ be a competitor for $\gamma_{\boldsymbol{F}}(G)$. Let $\left\{z_{k}\right\}$ be a sequence of points in $\mathbb{R}^{n}$ with

$$
\mathbb{R}^{n}=\bigcup_{k=1}^{\infty} B\left(z_{k}, 1 / 2\right) \quad \text { and } \quad \sum_{k=1}^{\infty} \chi_{B\left(z_{k}, 1\right)} \leq C,
$$

where $C$ depends only on $n$. Applying Lemma 8.7 we obtain

$$
\mathcal{H}_{h}\left(G \cap B\left(z_{k}, 1 / 2\right)\right) \leq C \int_{B\left(z_{k}, 1\right)}(\boldsymbol{F}(|u|)+\boldsymbol{F}(|\nabla u|)) d x
$$

for all $k$. Since $\mathcal{H}_{h}$ is countably subadditive it follows that

$$
\mathcal{H}_{h}(E) \leq \mathcal{H}_{h}(G) \leq C \int_{\mathbb{R}^{n}}(\boldsymbol{F}(|u|)+\boldsymbol{F}(|\nabla u|)) d x .
$$

By taking the infimum over all competitors $u$ we have $\mathcal{H}_{h}(E) \leq C \gamma_{\boldsymbol{F}}(G)$, and an appeal to Theorem 3.5(i) finishes the proof.

Before proceeding we state a simple symmetry formula concerning condition (7.1). It is based on the following application of Fubini's theorem.

Lemma 8.9. Suppose that $f, g:[0, \infty) \rightarrow[0, \infty)$ are nonincreasing functions such that

$$
s<f(t) \Rightarrow t \leq g(s) \text { and } t<g(s) \Rightarrow s \leq f(t) .
$$

(This is a weak form of $g$ being the inverse of $f$.) Then

$$
\int_{0}^{\infty} f(t) d t=\int_{0}^{\infty} g(s) d s .
$$

Proof. Using the Fubini theorem, from (8.3) we obtain

$$
\int_{0}^{\infty} f(t) d t=\int_{0}^{\infty}\left(\int_{0}^{f(t)} d s\right) d t=\int_{s<f(t)} d s d t
$$




$$
\leq \int_{t \leq g(s)} d s d t=\int_{0}^{\infty}\left(\int_{0}^{g(s)} d t\right) d s=\int_{0}^{\infty} g(s) d s
$$

and vice versa.

Proposition 8.10. Let $\boldsymbol{F}$ and $\boldsymbol{G}$ be $N$-functions. Then

$$
\int_{0}^{\infty}\left[\boldsymbol{G}^{\prime}\right]^{-1}\left(\frac{1}{\boldsymbol{F}^{\prime}(t)}\right) d t=\int_{0}^{\infty}\left[\boldsymbol{F}^{\prime}\right]^{-1}\left(\frac{1}{\boldsymbol{G}^{\prime}(t)}\right) d t
$$

Proof. Recall that $\boldsymbol{G}^{\prime}, \boldsymbol{F}^{\prime}$ are right-sided derivatives and their inverses are understood as in Definition 2.17. In view of Lemma 8.9 it is enough to verify (8.3) for

$$
f(t)=\left[\boldsymbol{G}^{\prime}\right]^{-1}\left(\frac{1}{\boldsymbol{F}^{\prime}(t)}\right) \text { and } g(s)=\left[\boldsymbol{F}^{\prime}\right]^{-1}\left(\frac{1}{\boldsymbol{G}^{\prime}(s)}\right) .
$$

If $s<f(t)$, then by Definition 2.17, $s$ belongs to the interval $\{\sigma>0$ : $\left.\boldsymbol{G}^{\prime}(\sigma) \leq 1 / \boldsymbol{F}^{\prime}(t)\right\}$. Hence $\boldsymbol{F}^{\prime}(t) \leq 1 / \boldsymbol{G}^{\prime}(s)$, which, again by Definition 2.17 , yields $t \leq g(s)$. The proof of the other implication is similar.

For the remainder of the section we specialize to a gauge $h$ of the form $h_{m}(r)=r^{n-m}$ for $1<m<n$ ( $m$ need not be an integer). The $(n-m)-$ dimensional Hausdorff content is defined by

$$
\mathcal{H}_{\infty}^{n-m}(E)=\boldsymbol{\alpha}(n-m) \mathcal{H}_{h_{m}}(E),
$$

where $\boldsymbol{\alpha}(k)$ is the normalizing constant of the $k$-dimensional Hausdorff measure, and the fractional maximal function $M_{m, R}$ is given by

$$
M_{m, R} \mu(x)=M_{h_{m}, R} \mu(x) .
$$

Proposition 8.11. Let $\boldsymbol{F}$ be an $N$-function. If $\mu$ is a Radon measure on $\mathbb{R}^{n}$ then

$$
W_{\boldsymbol{F}, R} \mu(x) \leq M_{m, R} \mu(x)^{1 /(m-1)} \int_{0}^{\infty}\left(\boldsymbol{F}^{\prime}(t)\right)^{1 /(1-m)} d t
$$

for all $x \in \mathbb{R}^{n}$.

Proof. Define $\boldsymbol{G}(r)=m^{-1} r^{m}$. Then $\boldsymbol{G}$ is an $N$-function and, by Proposition 8.10,

$$
\begin{aligned}
\int_{0}^{\infty}\left(\boldsymbol{F}^{\prime}(t)\right)^{1 /(1-m)} d t & =\int_{0}^{\infty}\left[\boldsymbol{G}^{\prime}\right]^{-1}\left(\frac{1}{\boldsymbol{F}^{\prime}(t)}\right) d t=\int_{0}^{\infty}\left[\boldsymbol{F}^{\prime}\right]^{-1}\left(\frac{1}{\boldsymbol{G}^{\prime}(t)}\right) d t \\
& =\int_{0}^{\infty}\left[\boldsymbol{F}^{\prime}\right]^{-1}\left(t^{1-m}\right) d t
\end{aligned}
$$


By the change of variable $r=M_{m, R} \mu(x)^{1 /(m-1)} t$, this implies

$$
\begin{aligned}
W_{\boldsymbol{F}, R} \mu(x) & =\int_{0}^{R}\left[\boldsymbol{F}^{\prime}\right]^{-1}\left(\frac{\mu(B(x, r))}{r^{n-1}}\right) d r \leq \int_{0}^{R}\left[\boldsymbol{F}^{\prime}\right]^{-1}\left(\frac{M_{m, R} \mu(x) r^{n-m}}{r^{n-1}}\right) d r \\
& \leq M_{m, R} \mu(x)^{1 /(m-1)} \int_{0}^{\infty}\left[\boldsymbol{F}^{\prime}\right]^{-1}\left(t^{1-m}\right) d t \\
& =M_{m, R} \mu(x)^{1 /(m-1)} \int_{0}^{\infty}\left(\boldsymbol{F}^{\prime}(t)\right)^{1 /(1-m)} d t . \text { - }
\end{aligned}
$$

THEOREM 8.12. Let $\boldsymbol{F}$ be a Young function. For an arbitrary set $E \subset \mathbb{R}^{n}$ we have

$$
\mathcal{H}_{\infty}^{n-m}(E) \leq C \gamma_{\boldsymbol{F}}(E)\left(\int_{0}^{\infty}\left(\boldsymbol{F}^{\prime}(t)\right)^{1 /(1-m)} d t\right)^{m-1},
$$

where $C$ depends only on $n$ and $m$.

Proof. The proof follows the lines of those of Lemma 8.6 and Theorem 8.8 but we must pay more attention to the constants. Assume that

$$
\int_{0}^{\infty}\left(\boldsymbol{F}^{\prime}(t)\right)^{1 /(1-m)} d t<\infty
$$

With $\boldsymbol{G}(r)=m^{-1} r^{m}$, this may be written as

$$
\int_{0}^{\infty}\left[\boldsymbol{G}^{\prime}\right]^{-1}\left(\frac{1}{\boldsymbol{F}^{\prime}(t)}\right) d t<\infty
$$

Applying Theorem 7.2 we may replace $\boldsymbol{F}$ by a strictly convex continuously differentiable $N$-function $\boldsymbol{F}_{1}$ satisfying $\Delta_{2}$ and $\nabla_{2}$ globally such that $\boldsymbol{F}_{1} \leq \boldsymbol{F}$ and

$$
\int_{0}^{\infty}\left[\boldsymbol{G}^{\prime}\right]^{-1}\left(\frac{1}{\boldsymbol{F}_{1}^{\prime}(t)}\right) d t \leq C \int_{0}^{\infty}\left[\boldsymbol{G}^{\prime}\right]^{-1}\left(\frac{1}{\boldsymbol{F}^{\prime}(t)}\right) d t .
$$

Observe that this implies $\gamma_{\boldsymbol{F}_{1}}(E) \leq \gamma_{\boldsymbol{F}}(E)$ for all $E \subset \mathbb{R}^{n}$. By Proposition 8.10 ,

$$
\begin{aligned}
\int_{0}^{\infty}\left[\boldsymbol{F}_{1}^{\prime}\right]^{-1}\left(\frac{h_{m}(r)}{r^{n-1}}\right) d r & =\int_{0}^{\infty}\left[\boldsymbol{F}_{1}^{\prime}\right]^{-1}\left(\frac{1}{\boldsymbol{G}^{\prime}(r)}\right) d r \\
& =\int_{0}^{\infty}\left[\boldsymbol{G}^{\prime}\right]^{-1}\left(\frac{1}{\boldsymbol{F}_{1}^{\prime}(t)}\right) d t<\infty,
\end{aligned}
$$

so $\boldsymbol{F}_{1}$ satisfies (8.1). Proceeding as in the proofs of Lemmas 8.6 and 8.7, if $B=B(z, 1)$ and $G \subset B(z, 1 / 2)$ is an open set, we have $1 \leq C W_{F_{1}, 4} \mu(x)$ for almost all $x \in G$. Here the constant $C$ depends on $n$ and the $\Delta_{2}$ and 
$\nabla_{2}$ constants of $\boldsymbol{F}_{1}$, which in turn depend on the corresponding constants of $\boldsymbol{G}$. Thus $C$ depends only on $m$ and $n$. The previous proposition implies

$$
W_{\boldsymbol{F}_{1}, 4} \mu(x) \leq C M_{m, 4} \mu(x)^{1 /(m-1)} \int_{0}^{\infty}\left(\boldsymbol{F}^{\prime}(t)\right)^{1 /(1-m)} d t,
$$

so we obtain the lower bound

$$
M_{m, 4} \mu(x) \geq C\left(\int_{0}^{\infty}\left(\boldsymbol{F}^{\prime}(t)\right)^{1 /(1-m)} d t\right)^{1-m}
$$

at all points of $G$. Proposition 8.2 and Lemma 6.4 imply

$$
\mathcal{H}_{h_{m}}(G) \leq C\left(\int_{0}^{\infty}\left(\boldsymbol{F}^{\prime}(t)\right)^{1 /(1-m)} d t\right)^{m-1}{ }_{\mathrm{c}_{\boldsymbol{F}}}(G, B) .
$$

Repeating the argument in the proofs of Lemma 8.7 and Theorem 8.8 with (8.5) in place of (8.2) we obtain

$$
\mathcal{H}_{h_{m}}(E) \leq C \gamma_{\boldsymbol{F}_{1}}(E)\left(\int_{0}^{\infty}\left(\boldsymbol{F}^{\prime}(t)\right)^{1 /(1-m)} d t\right)^{m-1}
$$

for any $E \subset \mathbb{R}^{n}$, completing the argument.

Finally we arrive at the result needed in [MSZ].

THEOREM 8.13. Suppose that $m>1$ and $\boldsymbol{F}$ is a Young function satisfying

$$
\int_{0}^{\infty}\left(\boldsymbol{F}^{\prime}(t)\right)^{1 /(1-m)} d t \leq 1 .
$$

Let $E \subset \mathbb{R}^{n}$. If $u \in W_{\text {loc }}^{1,1}\left(\mathbb{R}^{n}\right)$ is precisely represented and $u \geq 1$ on $E$, then

$$
\mathcal{H}_{\infty}^{n-m}(E) \leq C \int_{\mathbb{R}^{n}}(\boldsymbol{F}(|u|)+\boldsymbol{F}(|\nabla u|)) d x
$$

Proof. We may assume that the right-hand side of (8.6) is finite. By Corollary 4.7, $u$ has an $\boldsymbol{F}$-quasicontinuous representative, so the result follows from Theorems 4.8 and 8.12.

In the next section we will characterize those Young functions $\boldsymbol{F}$ satisfying the hypothesis of Theorem 8.13.

9. Lorentz and Orlicz spaces. In this section we prove some relations between Lorentz and Orlicz spaces. This is a generalization of results in $[\mathrm{KKM}]$ and $[\mathrm{CP}]$. We use an idea of simplification of proofs due to Cianchi (private communication). 
DeFinition 9.1. Let $f$ be a measurable function defined on a set $\Omega \subset \mathbb{R}^{n}$. The distribution function of $f$ is given by

$$
\mu_{f}(s)=\{x \in \Omega:|f(x)|>s\} .
$$

For the rest of this section we consider an $N$-function $\Phi$ satisfying $\Delta_{2}$ globally and define $\boldsymbol{G}$ by

$$
1 / \boldsymbol{G}(t)=\Phi(1 / t), \quad t>0,
$$

with the understanding that $\boldsymbol{G}(0)=0$. Thus $\boldsymbol{G}$ is an $N$-function satisfying $\Delta_{2}$ globally.

Definition 9.2. Let $\Omega \subset \mathbb{R}^{n}$ be an open set. If $f$ is a measurable function on $\Omega$ we define

$$
\|f\|_{L_{\Phi, 1}(\Omega)}:=\int_{0}^{\infty} \boldsymbol{G}^{-1}\left(\mu_{f}(s)\right) d s .
$$

The Lorentz space $L_{\Phi, 1}(\Omega)$ is defined as

$$
L_{\Phi, 1}(\Omega)=\left\{f \in L_{\mathrm{loc}}^{1}(\Omega):\|f\|_{L_{\Phi, 1}(\Omega)}<\infty\right\} .
$$

Since $\Phi$ satisfies $\Delta_{2}$ globally, $\|\cdot\|_{L_{\Phi, 1}(\Omega)}$ is a norm under which $L_{\Phi, 1}(\Omega)$ is a Banach space.

Lemma 9.3. Suppose that $f \in L_{\Phi, 1}(\Omega),\|f\|_{L_{\Phi, 1}(\Omega)}=1$. Then there exists a Young function $\boldsymbol{F}$ satisfying

$$
\int_{0}^{\infty}\left[\boldsymbol{G}^{\prime}\right]^{-1}\left(\frac{1}{\boldsymbol{F}^{\prime}(t)}\right) d t=1
$$

and

$$
\int_{\Omega} \boldsymbol{F}(|f(x)|) d x \leq 1 .
$$

Proof. We may assume that $f \geq 0$. Define

$$
\boldsymbol{F}(t)=\int_{0}^{t}\left(\boldsymbol{G}^{-1}\right)^{\prime}\left(\mu_{f}(s)\right) d s .
$$

It is routine to verify that $\boldsymbol{F}$ is a Young function. It follows at once that

$$
\begin{aligned}
\int_{0}^{\infty}\left[\boldsymbol{G}^{\prime}\right]^{-1}\left(\frac{1}{\boldsymbol{F}^{\prime}(t)}\right) d t & =\int_{0}^{\infty}\left[\boldsymbol{G}^{\prime}\right]^{-1}\left(\frac{1}{\left(\boldsymbol{G}^{-1}\right)^{\prime}\left(\mu_{f}(t)\right)}\right) d t \\
& =\int_{0}^{\infty}\left[\boldsymbol{G}^{\prime}\right]^{-1}\left(\boldsymbol{G}^{\prime}\left(\boldsymbol{G}^{-1}\left(\mu_{f}(t)\right)\right)\right) d t \\
& =\int_{0}^{\infty} \boldsymbol{G}^{-1}\left(\mu_{f}(t)\right) d t=\|f\|_{L_{\Phi, 1}(\Omega)}=1 .
\end{aligned}
$$


Since $\boldsymbol{G}$ is convex and increasing, and $\boldsymbol{G}(0)=0$, the inverse of $\boldsymbol{G}$ is a concave function satisfying

$$
\left(\boldsymbol{G}^{-1}\right)^{\prime}(\lambda) \leq \frac{\boldsymbol{G}^{-1}(\lambda)-\boldsymbol{G}^{-1}(0)}{\lambda-0}=\frac{\boldsymbol{G}^{-1}(\lambda)}{\lambda}
$$

and thus

$$
\boldsymbol{F}^{\prime}(t) \leq \frac{\boldsymbol{G}^{-1}\left(\mu_{f}(t)\right)}{\mu_{f}(t)} .
$$

It follows by an application of Fubini's theorem that

$$
\begin{aligned}
\int_{\Omega} \boldsymbol{F}(f(x)) d x & =\int_{0}^{\infty} \boldsymbol{F}^{\prime}(t) \mu_{f}(t) d t \\
& \leq \int_{0}^{\infty} \frac{\boldsymbol{G}^{-1}\left(\mu_{f}(t)\right)}{\mu_{f}(t)} \mu_{f}(t) d t=\int_{0}^{\infty} \boldsymbol{G}^{-1}\left(\mu_{f}(t)\right) d t=\|f\|_{L_{\Phi, 1}(\Omega)}=1 .
\end{aligned}
$$

Lemma 9.4. Suppose that $f$ is a measurable function on $\Omega$ and that $\boldsymbol{F}$ is a Young function satisfying (9.1) and (9.2). Then $f \in L_{\Phi, 1}(\Omega)$ and

$$
\|f\|_{L_{\Phi, 1}(\Omega)} \leq 2 .
$$

Proof. We may assume that $f \geq 0$. Let $\widetilde{\boldsymbol{G}}$ be the Young function complementary to $\boldsymbol{G}$. By convexity we have

$$
\widetilde{\boldsymbol{G}}(\lambda) \leq \lambda \widetilde{\boldsymbol{G}}^{\prime}(\lambda) \text { for all } \lambda>0 .
$$

Then Young's inequality (2.1) implies

$$
\boldsymbol{G}^{-1}\left(\mu_{f}(t)\right) \frac{1}{\boldsymbol{F}^{\prime}(t)} \leq \mu_{f}(t)+\widetilde{\boldsymbol{G}}\left(\frac{1}{\boldsymbol{F}^{\prime}(t)}\right) \leq \mu_{f}(t)+\frac{1}{\boldsymbol{F}^{\prime}(t)} \widetilde{\boldsymbol{G}}^{\prime}\left(\frac{1}{\boldsymbol{F}^{\prime}(t)}\right) .
$$

Multiplying both sides by $\boldsymbol{F}^{\prime}(t)$ and integrating we obtain

$$
\begin{aligned}
\|f\|_{L_{\Phi, 1}(\Omega)} & =\int_{0}^{\infty} \boldsymbol{G}^{-1}\left(\mu_{f}(t)\right) d t \\
& \leq \int_{0}^{\infty} \boldsymbol{F}^{\prime}(t) \mu_{f}(t) d t+\int_{0}^{\infty} \widetilde{\boldsymbol{G}}^{\prime}\left(\frac{1}{\boldsymbol{F}^{\prime}(t)}\right) d t \\
& =\int_{\Omega} \boldsymbol{F}(f(x)) d x+\int_{0}^{\infty}\left[\boldsymbol{G}^{\prime}\right]^{-1}\left(\frac{1}{\boldsymbol{F}^{\prime}(t)}\right) d t \leq 2 .
\end{aligned}
$$

Combining the preceding lemmas, we can show that $L_{\Phi, 1}(\Omega)$ may be written as a union of Orlicz spaces.

Theorem 9.5.

$$
L_{\Phi, 1}(\Omega)=\bigcup\left\{L^{\boldsymbol{F}}(\Omega): \boldsymbol{F} \text { satisfies }(9.1)\right\} .
$$


Proof. If $f \in L_{\Phi, 1}(\Omega)$, then $\tilde{f}=f /\|f\|_{L_{\Phi, 1}(\Omega)}$ satisfies $\|\widetilde{f}\|_{L_{\Phi, 1}(\Omega)}=1$. By Lemma 9.3 there is a Young function $\boldsymbol{F}$ for which (9.1) holds and for which $\widetilde{f} \in L^{\boldsymbol{F}}(\Omega)$, hence $f \in L^{\boldsymbol{F}}(\Omega)$.

If $f \in L^{\boldsymbol{F}}(\Omega)$ for a Young function $\boldsymbol{F}$ satisfying $(9.1)$, then $\widetilde{f}=f /\|f\|_{L^{\boldsymbol{F}}(\Omega)}$ satisfies (9.2). Lemma 9.4 implies that $\widetilde{f} \in L_{\Phi, 1}(\Omega)$, hence $f \in L_{\Phi, 1}(\Omega)$.

The Lorentz space $L^{m, 1}(\Omega)$ is defined as $L_{\Phi, 1}(\Omega)$ corresponding to $\Phi(r)=$ $\boldsymbol{G}(r)=r^{m}$. In light of Theorems 9.5 and 7.2 we have the following.

Corollary 9.6. Let $m>1$. Then

$L^{m, 1}(\Omega)=\bigcup\left\{L^{\boldsymbol{F}}(\Omega): \boldsymbol{F}\right.$ is a Young function with $\left.\int_{0}^{\infty}\left(\boldsymbol{F}^{\prime}(t)\right)^{1 /(1-m)} d t=1\right\}$.

\section{References}

[AH] D. R. Adams and L. I. Hedberg, Function Spaces and Potential Theory, Springer, 1996.

[A] R. A. Adams, Sobolev Spaces, Academic Press, 1975.

[Ai] N. Aïssaoui, Maximal operators, Lebesgue points and quasicontinuity in strongly nonlinear potential theory, Acta Math. Univ. Comenian. 71 (2002), 35-50.

[BZ] T. Bagby and W. P. Ziemer, Pointwise differentiability and absolute continuity, Trans. Amer. Math. Soc. 191 (1974), 129-148.

[BL] T. Bhattacharya and F. Leonetti, A new Poincaré inequality and its application to the regularity of minimizers of integral functions with nonstandard growth, Nonlinear Anal. 17 (1991), 833-839.

[BO] J. Björn and J. Onninen, Orlicz capacities and Hausdorff measures on metric spaces, Math. Z. 251 (2005), 131-146.

[C1] A. Cianchi, Boundedness of solutions to variational problems under general growth conditions, Comm. Partial Differential Equations 22 (1997), 1629-1646.

[C2] - Strong and weak type inequalities for some classical operators in Orlicz spaces, J. London Math. Soc. (2) 60 (1999), 187-202.

[CP] A. Cianchi and L. Pick, Sobolev embeddings into BMO, VMO, and $L_{\infty}$, Ark. Mat. 36 (1998), 317-340.

[CFL] S. Conti, I. Fonseca and G. Leoni, A $\Gamma$-convergence result for the two-gradient theory of phase transitions, Scientific Report 01-CNA-008, Carnegie Mellon Univ., 2001.

[DG] E. De Giorgi, Teoremi di semicontinuità nel calcolo delle variazioni, Istit. Naz. Alta Mat., Roma, 1968/69.

[FZ] H. Federer and W. P. Ziemer, The Lebesgue set of a function whose distribution derivatives are -th power summable, Indiana Univ. Math. J. 22 (1972), 139-158.

[FP] A. Fiorenza and A. Prignet, Orlicz capacities and applications to some existence question for elliptic PDEs having measure data, ESAIM Control Optim. Calc. Var. 9 (2003), 317-341.

[F] O. Frostman, Potentiel d'équilibre et capacité des ensembles avec quelques applications à la théorie des fonctions, Medd. Lunds Univ. Mat. Sem. 3 (1935), $1-118$. 
[KKM] J. Kauhanen, P. Koskela and J. Malý, On functions with derivatives in a Lorentz space, Manuscripta Math. 100 (1999), 87-101.

[M1] J. Malý, Wolff potential estimates for minimizers of Orlicz type Dirichlet integrals, ibid. 110 (2003), 513-525.

[M2] - Coarea properties of Sobolev functions, in: Function Spaces, Differential Operators and Nonlinear Analysis (Teistungen, 2001), Birkhäuser, 2003, 371381.

[M3] - Coarea integration in metric spaces, in: Nonlinear Analysis, Function Spaces and Applications, Vol. 7, Proc. Spring School Held in Prague, July 17-22, 2002, B. Opic and J. Rákosník (eds.), Math. Inst. Acad. Sci. of the Czech Republic, Praha, 2003, 142-192.

[MSZ] J. Malý, D. Swanson and W. P. Ziemer, The coarea formula for Sobolev mappings, Trans. Amer. Math. Soc. 355 (2003), 477-492.

[MZ] J. Malý and W. P. Ziemer, Fine Regularity of Solutions of Elliptic Partial Differential Equations, Math. Surveys Monogr. 51, Amer. Math. Soc., 1997.

[Ma1] V. G. Maz'ya, The p-conductivity and theorems on imbedding certain function spaces into a C-space, Dokl. Akad. Nauk SSSR 140 (1961), 299-302 (in Russian); English transl.: Soviet Math. Dokl. 2 (1961), 1200-1203.

[Ma2] V. G. Maz'ya, Sobolev Spaces, Springer, Berlin, 1985.

[MH] V. G. Maz'ya and V. P. Havin [V. P. Khavin], Nonlinear potential theory, Uspekhi Mat. Nauk 27 (1972), no. 6, 67-138 (in Russian); English transl.: Russian Math. Surveys 27 (1972), no. 6, 71-148.

[Me] N. Meyers, A theory of capacities for potentials of functions in Lebesgue classes, Math. Scand. 26 (1970), 255-292.

[RR] M. M. Rao and Z. D. Ren, Theory of Orlicz Spaces, Monographs Textbooks Pure Appl. Math. 146, Dekker, 1991.

[Re] Yu. G. Reshetnyak, On the concept of capacity in the theory of functions with generalized derivatives, Sibirsk. Mat. Zh. 10 (1969), 1109-1138 (in Russian); English transl.: Siberian Math. J. 10 (1969), 818-842.

[T] H. Tuominen, Orlicz-Sobolev spaces on metric measure spaces, Ann. Acad. Sci. Fenn. Math. Diss. 135 (2004), 86 pp.

[Z] W. P. Ziemer, Weakly Differentiable Functions, Springer, 1989.

Faculty of Mathematics and Physics

Charles University-KMA

Sokolovská 83

18675 Praha 8, Czech Republic

and

PřF UJEP

40096 Ústí n. L., Czech Republic

E-mail: maly@karlin.mff.cuni.cz

Department of Mathematics

Indiana University

Bloomington, IN 47405, U.S.A.

E-mail: ziemer@indiana.edu
Department of Mathematics University of Louisville Louisville, KY 40292, U.S.A. E-mail: david.swanson@louisville.edu 